\title{
Dual Mission Scenarios for the Human Lunar Campaign - Performance, Cost and Risk Benefits
}

\author{
Rudolph J. Saucillo, NASA Langley Research Center, Rudolph.J.Saucillo@nasa.gov, 757-864-7224 \\ David M. Reeves, NASA Langley Research Center, David.M.Reeves@nasa.gov, 757-864-9256 \\ Jonathan D. Chrone, Analytical Mechanics Associates, Inc., Jonathan.D.Chrone@nasa.gov, 757-864-8043 \\ Chel Stromgren, SAIC, Inc., Chel.Stromgren@saic.com, 703-676-6379 \\ John D. Reeves, NASA Langley Research Center, John.D.Reeves@nasa.gov, 757-864-4510 \\ David D. North, NASA Langley Research Center, David.N.North@nasa.gov, 757-864-7285 \\ 1 North Dryden Street, Mail Stop 462 \\ Hampton, VA 23681-2199
}

Abstract-NASA's future human lunar campaign faces significant performance, cost and risk challenges. These include:

o Providing the capability to access large portions of the lunar surface for expanded science and exploration within the performance constraints of the integrated transportation system;

o Minimizing the annual cost of the lunar campaign; and

o Minimizing operational risk including probability of loss of mission (PLOM) and probability of loss of crew (PLOC)

Innovative lunar operations scenarios which address these challenges are potentially feasible based on the concept of dual, sequential missions utilizing a common crew and a single Ares I/Crew Exploration Vehicle (CEV). Dual mission scenarios possible within the scope of baseline technology planning include Outpost-based sortie missions, dual sortie missions, and enhanced Outpost deployment. Additional mission scenarios are potentially possible with the development of advanced capabilities. These include abort to the lunar surface options and Lander reusability options.

Top-level benefits of these dual mission scenarios may be estimated by comparison to the Constellation Program reference two-mission-per-year lunar campaign. The primary performance benefit is substantially improved surface access and an increase in payload mass to the lunar surface for Mission B of the two mission sequence. The cost benefit is the accomplishment of Mission B with a "single launch solution" since no Ares I launch is required. Compared with other single launch solutions which have been proposed, this option provides an additional cost benefit since it does not require the human rating of the Ares V. Probability of loss of mission for dual mission scenarios is significantly lower since Mission B does not require the successful launch of two launch vehicles and an orbital rendezvous in time for the translunar injection window. Cumulative risk to the crew is lower since crew exposure to Earth launch risks and Earth entry risks is reduced versus comparable Constellation Program reference two-mission-per-year scenarios.-

\section{TABLE OF CONTENTS}

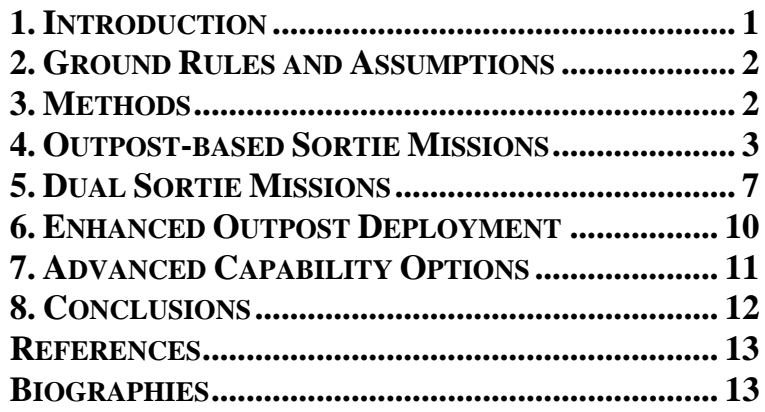

\section{INTRODUCTION}

The Constellation Program is currently defining a lunar architecture and associated capabilities to establish a lunar South Pole Outpost beginning in 2019. In addition to deploying and supporting the permanently crewed South Pole Outpost, this lunar architecture is required to have the capability to accommodate sorties to any location on the lunar surface in order to maximize opportunities for science and exploration [1].

The recently completed study by the NASA Lunar Architecture Team (LAT) [2] showed that requirements for global sortie missions - as opposed to South Pole Outpost deployment and support - are the drivers for architecture performance. Specifically, performance constraints imposed by the integrated transportation system including the Ares I and Ares V launch vehicles, Earth Departure Stage (EDS), and Lunar Lander may limit sortie capability.

The dual mission scenarios described below ${ }^{12}$ were developed as part of the LAT study as a potential means to optimize architecture performance. The primary goal was to define an operations concept for a cost effective, robust human sortie capability providing expanded science opportunities beyond the lunar Outpost. A secondary goal was to define an option to deliver and deploy substantial lunar Outpost cargo within the specified capabilities of the integrated transportation system.

\footnotetext{
${ }^{1}$ U.S. Government work not protected by U.S. copyright.

2 IEEEAC paper\#1183, Version 7, Updated 2007:12:06
} 


\section{GROUND RULES AND ASSUMPTIONS}

The Lunar Lander concept used in this analysis is based on the following:

o Design Analysis Cycle \#1 (LDAC \#1) Lander configuration provided by the Constellation Program Lunar Lander Project Office. This is a conceptual, early design cycle lander and does not represent a Lunar Lander Project Office final design.

o Lander Ascent Module with $8.2 \mathrm{~m}^{3}$ total internal volume and $6 \mathrm{~m}^{3}$ habitable volume

o 3 x $15 \mathrm{klb}$ thrust $\mathrm{LOX} / \mathrm{H}_{2}$ Lander Descent Module engines o $889 \mathrm{~m} / \mathrm{s}$ lunar orbit insertion (LOI) delta-V for the South

Pole Outpost

o 2,100 m/s lunar descent delta- $\mathrm{V}$ for all sites

o Up to 7 day low lunar orbit loiter capability

o Lander sized to deliver $6 \mathrm{t}$ of cargo to the South Pole

Outpost with 4 crew and 24 hours of crew life support logistics

o 20\% growth margin and 20\% program manager's reserve

(PMR) added to Lander dry mass

This LDAC \#1 Lander was augmented with a sortie "kit" to accommodate a crew of 2 for a 7 day surface stay (figure 1). Augmentations include a Suitlock located in the Descent Module mid bay with a tunnel connecting the Ascent Module. In this configuration the sortie crew will live in the Ascent Module with limited additional habitable volume available in the Suitlock. EVA suits will be stored externally as part of the Suitlock configuration. Power and thermal system modifications were also necessary to operate anywhere on the lunar surface including the extreme thermal environment of the equatorial region. Other Lander modifications include the capability to scavenge residual Descent Module $\mathrm{LOX} / \mathrm{H}_{2}$ propellant to support 7 day fuel cell operations for Lander power. The launch mass of this Lander including the 2-crew sortie kit is $56 \mathrm{t}$.

\section{METHODS}

\section{Lander Sizing}

The LDAT \#1 Lander was modeled with the NASA Langley Research Center-developed Exploration Architecture Model for In-space and ETO (EXAMINE) sizing tool. This Lander model was developed by sizing Lander tanks and other subsystems to accommodate the $6 \mathrm{t}$ payload capability for the nominal 4 crew mission to the South Pole Outpost. The Lander sortie kit was sized using subsystem data developed by discipline experts as part of the LAT study.

\section{Lander Performance and Surface Accessibility Assessment}

Plane change capabilities of the Lander/CEV stack were determined using the EXAMINE Lander model on a per site basis. The LOI delta-V to achieve an "anytime return" lunar orbit was analyzed using Copernicus, a flight performance tool utilized by the Constellation Program [3]. Analyses to establish surface accessibility was then performed in the MATLAB ${ }^{\circledR}$ environment to determine the allowable landed payload to a grid of landing sites. Landing sites requiring a plane change greater than the limits set by EXAMINE for landed payload were excluded.

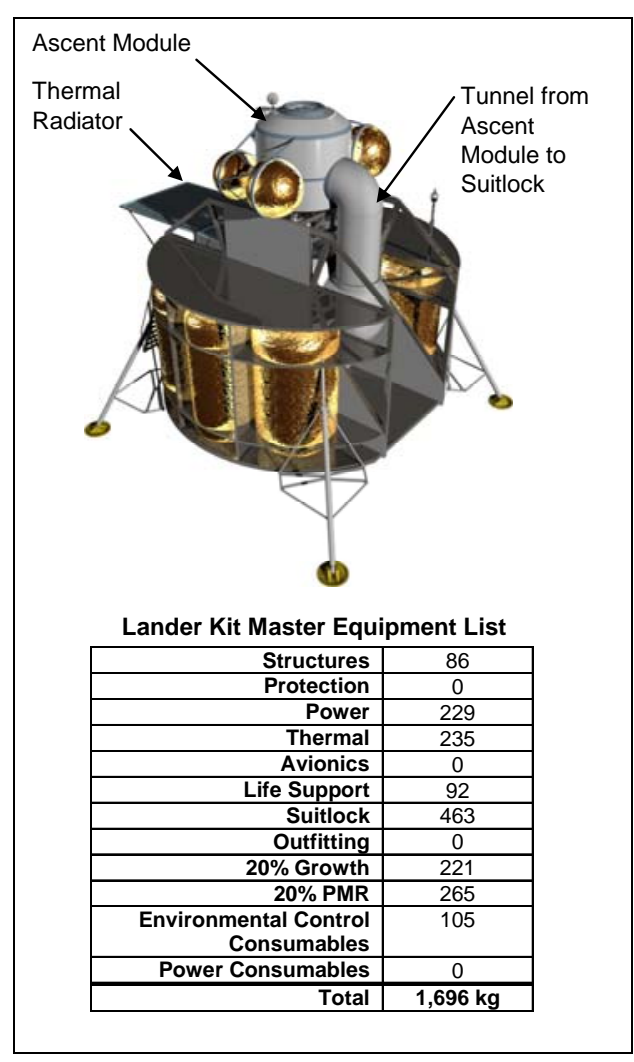

Figure 1 - Lunar Lander with sortie kit

\section{Cost Estimation}

Cost benefits of the dual mission scenarios were estimated by comparison to the author's interpretation of existing Constellation Program cost projections and estimates. Due to the sensitivity of current Agency projections and availability of data, quantitative comparison between scenarios is limited to normalized variable costs. A complete understanding of affordability impacts would require an analysis of all life cycle cost considerations, both fixed and variable.

\section{Risk Analysis}

Lunar mission PLOM and PLOC risks were evaluated using the risk module of the Probabilistic Campaign Assessment and Manifesting Tool (PCMAT). This Excel-based model predicts the risk of each stage of an integrated lunar mission based on primary risk drivers including engine selection. Risk estimates were developed using heritage data for legacy systems or similar technologies and were modified 
by the operational experience and reliability gained throughout a campaign.

\section{Modeling and Simulation}

The NASA Langley Research Center Synergistic Engineering Environment (SEE) was used to model and visualize the lunar orbital geometry for dual mission vehicle operations.

\section{OUtPost-BASEd SoRTiE Missions}

The Outpost-based sortie mission concept provides a means to access higher latitude regions near the lunar North and South Poles. These regions are generally of high scientific interest since solar wind volatiles are concentrated at these latitudes. Additionally, locations in this region including the South Pole Aitken Basin and the North Pole have been identified as priority science sites by the Lunar Exploration Analysis Group (LEAG) [4].

Figure 2 illustrates the operations concept for the Outpostbased sortie mission scenario. Mission A is similar to the Constellation Program reference mission for Outpost deployment. EDS \#1, Lander \#1, the CEV and four crew are launched by an Ares V and Ares I. The CEV performs a rendezvous and docks with the EDS \#1/Lander \#1 stack. EDS \#1 performs the translunar injection (TLI) maneuver and is expended. Lander \#1 performs LOI and the stack transfers to a low lunar polar orbit and loiters for up to 3 days. Lander \#1 and crew then descend to the South Pole Outpost site where the crew deploys Outpost infrastructure during a Mission A surface stay of up to 6 months. At the completion of Mission A, the crew returns to the CEV in low lunar polar orbit.

Mission B is initiated with an Ares V launch of EDS \#2 and Lander \#2 prior to completion of Mission A. Unlike Mission A, this mission is a direct launch to the moon since there is no requirement to rendezvous in low-Earth orbit. To optimize propellant usage between the EDS and Lander, EDS \#2 performs a portion of LOI utilizing the remainder of its propellant load. Lander \#2 then completes the LOI maneuver sequence and enters the CEV polar orbit. After the crew returns to the CEV and expends the Lander \#1 Ascent Module, the CEV performs a rendezvous and docks with Lander \#2.

To establish the proper orbit for the Mission B sortie site, Lander \#2 propulsion is utilized to maneuver the Lander \#2/CEV stack to the correct orbit plane. The crew loiters in lunar orbit for 24 hours to allow a navigation system update and then descends to the sortie site. For this analysis, it is assumed that two crew remain in the CEV and two crew descend to the lunar surface to accomplish Mission B. At the completion of a 4- to 7-day sortie, the crew returns to the CEV for return to Earth.

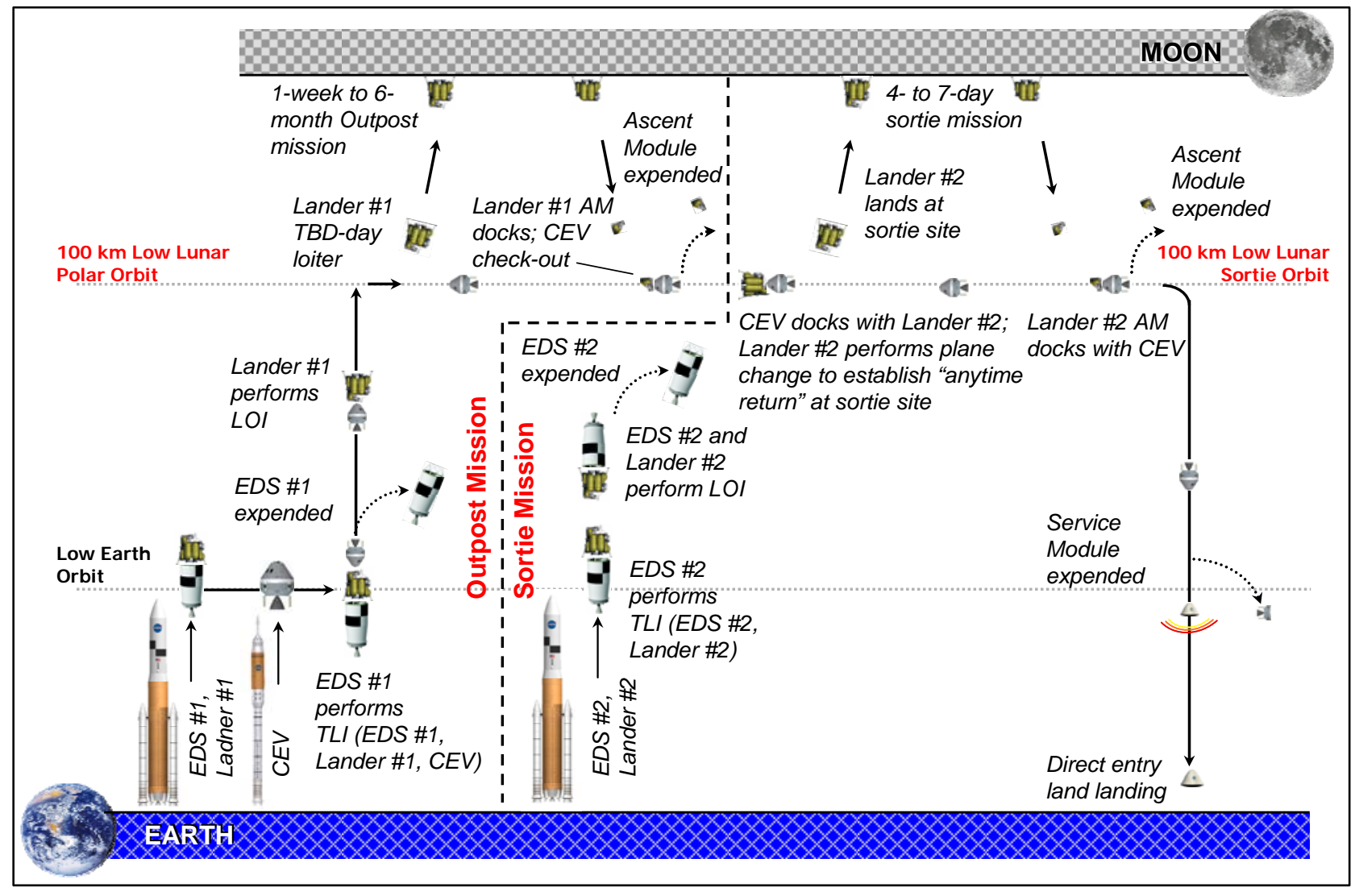

Figure 2 - Outpost-based sortie mission scenario 
Figure 3 illustrates the orbital geometry for a sortie mission from the South Pole Outpost. To begin the maneuver sequence, the crew performs a coplanar ascent from the Outpost to the CEV in polar orbit. The ascending node of this polar orbit may be set as part of the Mission A LOI maneuver or, alternately, may be established by waiting on the lunar surface for the desired ascending node prior to ascent. Both methods provide full longitudinal access for the Mission B sortie. The orbit for the Mission B sortie site is determined by the requirement for "anytime return" to the CEV from the lunar surface and the requirement for a coplanar descent to the lunar surface. To establish this orbit, a plane change is performed to put the CEV in a landing site-specific orbit which minimizes the wedge angle between the Mission B landing site and the orbit over the 7 day surface stay. Accessible latitudes are a function of lander performance and the minimum payload requirement.

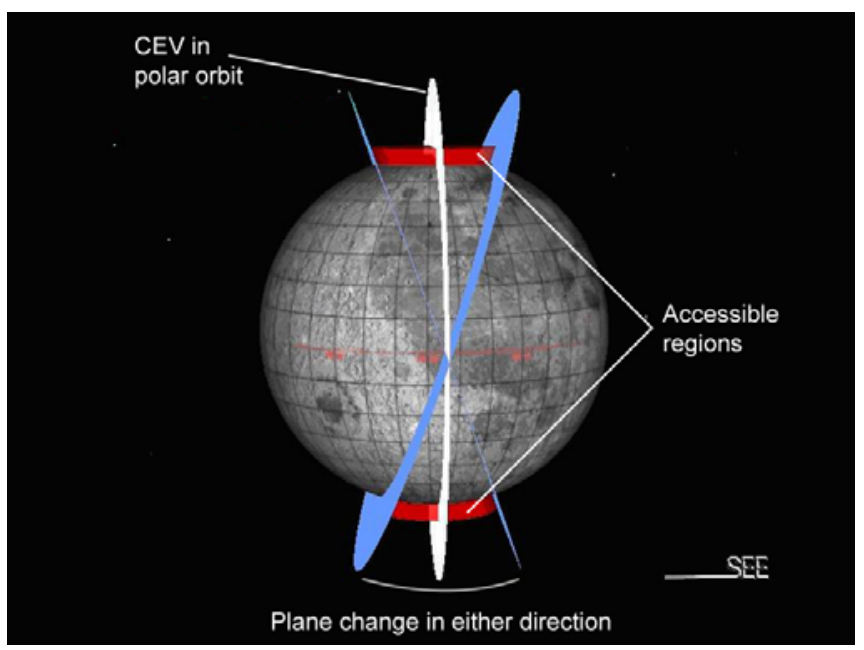

Figure 3 - Orbit plane change capability enabling Outpost-based sorties

The maneuvers performed by each element of the integrated transportation system are similar for Mission A and Mission B. The EDS in each mission performs two maneuvers. In Mission A the EDS performs a direct low-Earth orbit insertion maneuver and TLI; in Mission B the EDS performs TLI and a portion of LOI. In both missions the Lander Descent Module performs at least a portion of LOI and lunar descent.

\section{Performance Benefits}

Payload-to-the-lunar-surface capability is substantially increased in the Mission B sortie as a result of additional propellant available for Lander \#2 descent. This additional propellant is due to EDS \#2 transferring a smaller stack through TLI (i.e., Lander \#2 without a CEV) and using the remaining propellant to perform a portion of the LOI maneuver. Offloading a portion of the LOI maneuver onto EDS \#2 and "pushing" a smaller mass through the completion of LOI provides Lander \#2 with substantial additional propellant for lunar descent.
Higher latitude regions are accessible with the Outpostbased sortie mission scenario as illustrated in figure 4 . Payload-to-the-surface capability is a function of sortie site latitude based on Lander \#2 Descent Module propellant remaining after the plane change maneuver. That is, lower latitude sites require a greater Lander \#2/CEV plane change to establish anytime return. This results in a lower payload mass to the surface as sortie site latitude decreases. As shown in figure 4, a Lander with a 6 t payload capability to the South Pole Outpost with 4 crew and 24 hours of crew life support logistics for Mission A can deliver $8 \mathrm{t}$ of payload the North Pole ( $+88.6^{\circ}$ latitude) and $1 \mathrm{t}$ of payload to latitudes as low as $\pm 68^{\circ}$ with a crew of 2 for a 7 day mission.

\section{Cost Savings}

Top level cost benefits of the Outpost-based sortie scenario may be estimated by comparison to the Constellation Program reference two-mission-per-year lunar campaign. The primary cost benefit associated with the Outpost-based sortie scenario is the savings realized with one less Ares I/CEV stack launch per year. In comparison with other single launch solutions which have been proposed, this option provides an additional cost benefit since it does not require the human rating of the Ares $\mathrm{V}$.

Although the Ares I and CEV Project Office projections contain significant fixed costs that are not dependent on launch rate, there are flight rate-dependent variable/marginal costs associated with each system that relate to prime contractor materials and labor and associated government oversight. For Ares I these include variable costs of the upper stage, J-2 engine, and solid rocket booster/solid rocket motor first stage. For CEV these savings include the variable costs of the service module, launch abort system, and expendable heat shield. In addition to hardware and labor costs associated with the manufacture and delivery of the elements themselves, there are also savings associated with the ground and logistics processing of the individual CEV and Ares I vehicles and integrated stack.

Due to the sensitivity of current program estimates and projections as well as limitations on data sets, the cost analysis contained herein is limited to the variable costs associated with operating the various Constellation systems. A full understanding of the impacts associated with these dual mission scenarios requires a full life cycle analysis over the timeline of the campaign that includes both fixed, variable, and supporting operational costs for all related systems and overhead. Although variable cost savings are identified here, the fixed costs associated with these systems are typically the larger cost driver. Therefore, the results shown are demonstrative of the nature of the savings that may be realized and not the actual savings in context of the entire affordability picture. 


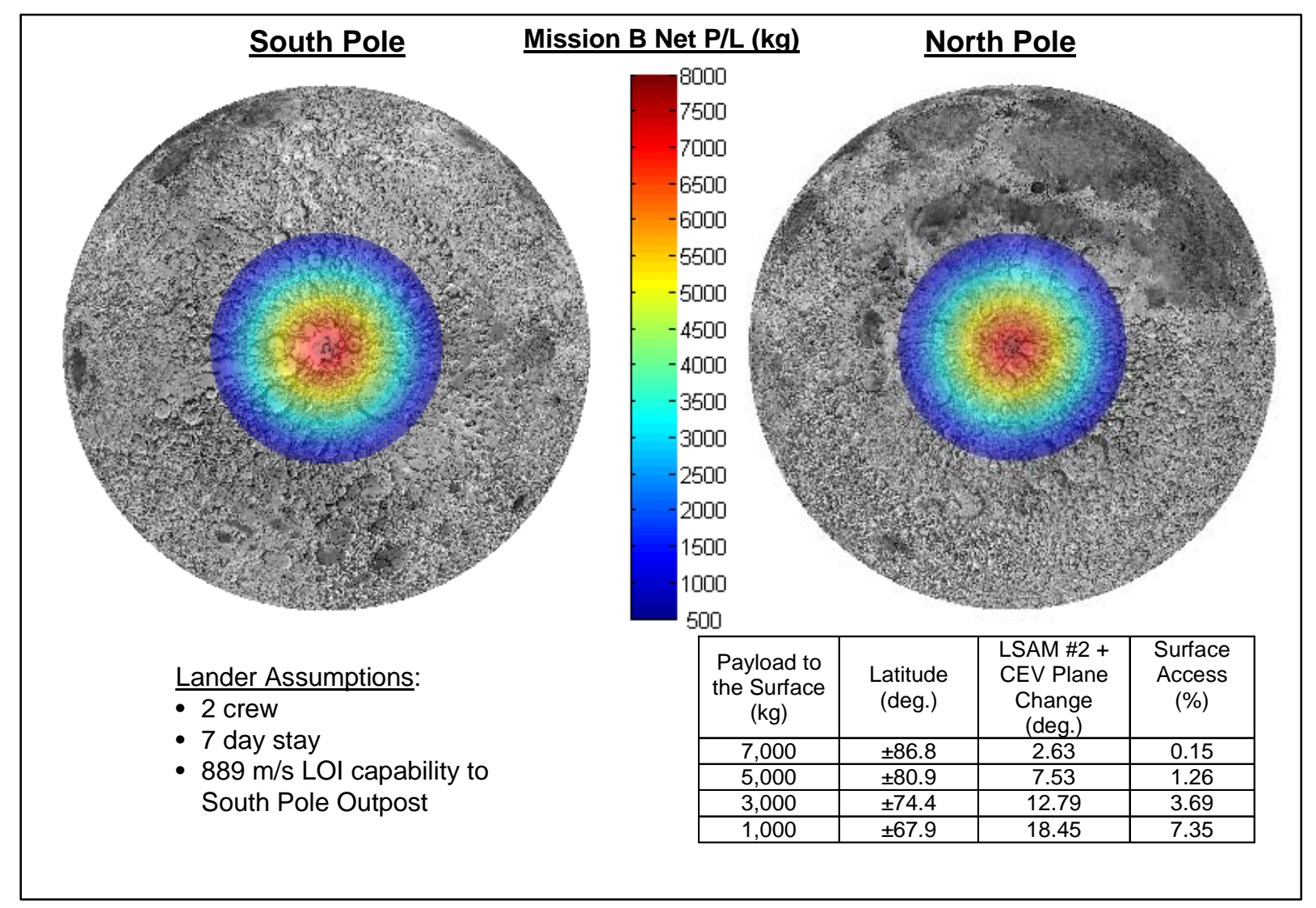

Figure 4 - Surface access for Outpost-based sortie missions

Figure 5 illustrates that the Outpost-based sortie scenario can lead to an annual variable savings of $\sim 8 \%$ versus the Constellation Program reference two-mission-per-year campaign. These savings are related to the variable contribution of one less Ares I/CEV flight in context of the variable costs of Ares I, Ares V, CEV, Lander, and Ground Operations. This estimate is based on the author's understanding of current Constellation Program estimates including certain assumptions with respect to limited data sets. These results indicate that the dual mission scenarios lead to a lower expected annual operating cost than the reference two independent missions per year lunar campaign.

\section{Risk Reduction}

Outpost-based sortie scenarios potentially offer a reduction in the overall PLOM and in the overall PLOC compared with the Constellation Program reference two-mission-peryear lunar campaign.

For purposes of this comparison, the evaluation of PLOM and PLOC risks was limited to the mission phases that are associated with the integrated transportation system. The five mission phases that were evaluated include: dual launch, ascent and lunar transfer, lunar descent and ascent, lunar rendezvous and docking, and return to Earth.
The dual launch risk captures the risk associated with launching the Ares I and Ares $\mathrm{V}$ in time to rendezvous in low-Earth orbit and perform TLI within the TLI window. This risk is a major contributor to PLOM but does not impact PLOC. The dual launch risk is also heavily impacted by vehicle maturity; the PLOM reduction predicted here might be even greater for early missions.

All risks in this analysis were evaluated assuming transportation system maturity. For PLOC, the maturity level of the Lander Ascent Module has a major impact on risk independent of scenario. However, the exposure to this risk does not change in this analysis since there are two ascent events in the dual mission scenarios and the Constellation Program two-mission-per-year reference. Therefore, the anticipated PLOC risk reductions for the dual mission scenarios are independent of Lander maturity while the absolute values in both scenarios are based on mature systems. For PLOM, lunar descent and ascent are major contributors to risk. However, as with PLOC, these events are not impacted in the dual mission scenarios.

Each of the dual mission scenarios provides additional options for return-to-Earth in the event of a CEV Service Module failure. The CEV Service Module will be checked out prior to crew descent to the lunar surface during Mission A. If the CEV Service Module has a major failure, the crew can return to Earth using Lander \#1 Descent 


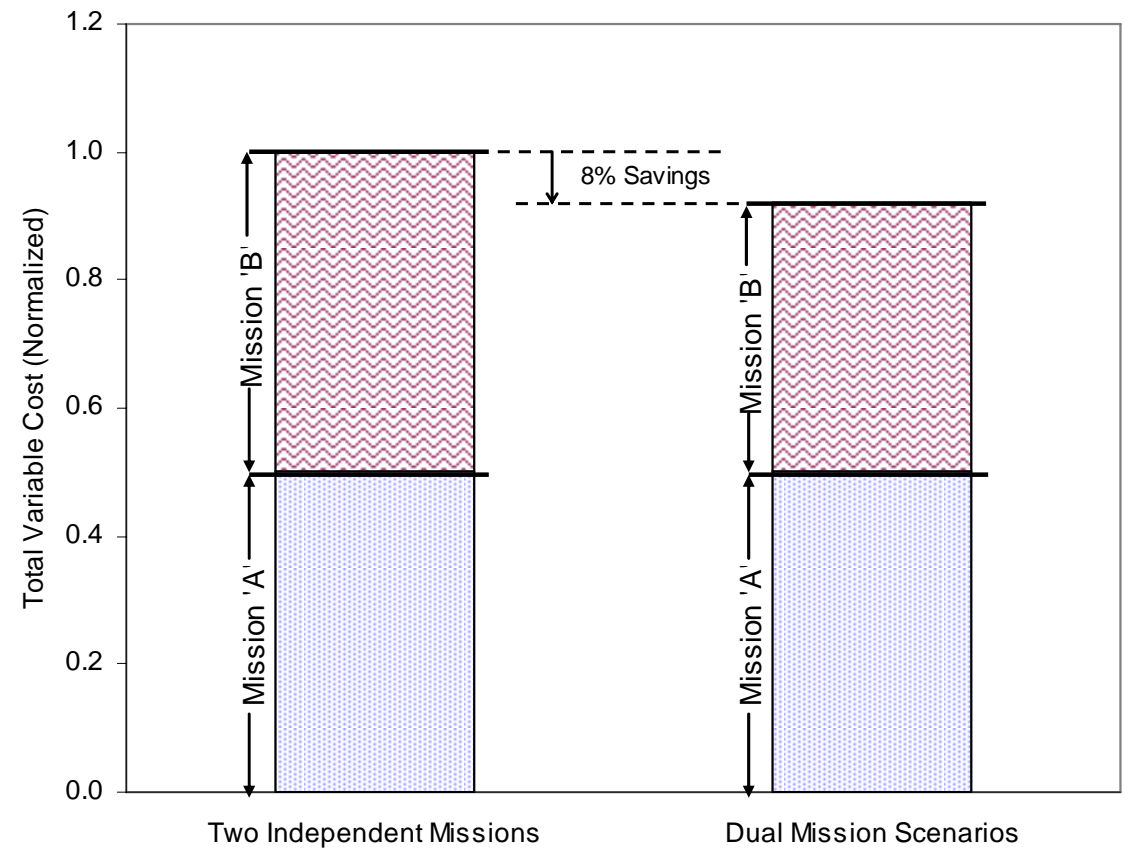

Figure 5 - Comparison of total variable costs (fixed costs not included)

Module propulsion. Since the cumulative probability of a CEV Service Module failure is higher after a multi-month exposure to the space environment (e.g., due to a micrometeoroid impact) the CEV Service Module will also be checked out after completion of Mission A when Lander \#2 is in lunar orbit. If the CEV Service Module is determined to have a major failure, Mission $\mathrm{B}$ will be aborted and the crew will return home using Lander \#2 Descent Module propulsion. The probability of a CEV Service Module failure is highly dependent on lunar orbit stay time. No assumptions have been made for CEV stay time in lunar orbit and the risk benefits for this additional return-to-Earth capability have not been modeled.

This risk analysis did not include an evaluation of surface operational risks for either surface stay activities or for the CEV in lunar orbit. These risks are highly dependent on the surface stay duration and are not impacted by the implementation of dual mission scenarios. The overall risk values presented, therefore, do not represent the total mission risk for either PLOC or PLOM. The total risks, including the surface stay risk, will be significantly greater than the values presented herein.

Probability of Loss of Mission-Dual mission scenarios can provide a significant risk reduction in the integrated transportation system PLOM components as shown in figure 6. For the baseline lunar mission, the total PLOM for the transportation phases of the mission, including the two launch risk, is $13.8 \%$. The total PLOM across two crewed missions to the lunar surface is $27.6 \%$. A dual mission scenario reduces the overall PLOM for two crewed landings to $18.2 \%$. This is an effective $34 \%$ reduction in the integrated transportation system PLOM components relative to the Constellation Program two-mission-per-year reference.

The Outpost-based sortie scenario eliminates several phases from the overall PLOM. Most significantly, this scenario requires only a single two-vehicle launch event, eliminating a dual launch risk of $8.6 \%$. In addition, the dual Outpostbased sortie scenario also eliminates an Earth return risk of $0.4 \%$ and reduces the risk of the second ascent and lunar transfer event by $0.6 \%$ through elimination of the second Ares I launch. The only addition to PLOM comes from the additional lunar docking and rendezvous event which increases PLOM by $0.3 \%$.

The reduction in PLOM could become an important factor from a campaign analysis context. Because loss of mission events have the potential to impart delays in the progress of the lunar campaign, the value of PLOM is critical to overall campaign success. A reduction in the PLOM across two missions of $9.4 \%$ out of $27.6 \%$, or an effective $34 \%$ reduction of the integrated transportation system PLOM components versus the reference, represents a very significant improvement in the expected loss of mission.

Probability of Loss of Crew-Figure 7 shows a comparison of the integrated transportation system PLOC components for an Outpost-based sortie scenario and the Constellation Program reference two-mission-per-year campaign. For a Constellation Program baseline lunar mission, the total PLOC for the transportation phases of the mission is $0.97 \%$. Multiplying by 2, the total PLOC for two crewed landings on the lunar surface is $1.94 \%$. The Outpost-based sortie scenarios reduce the integrated transportation system PLOC components for two crewed landings to $1.64 \%$ resulting in 


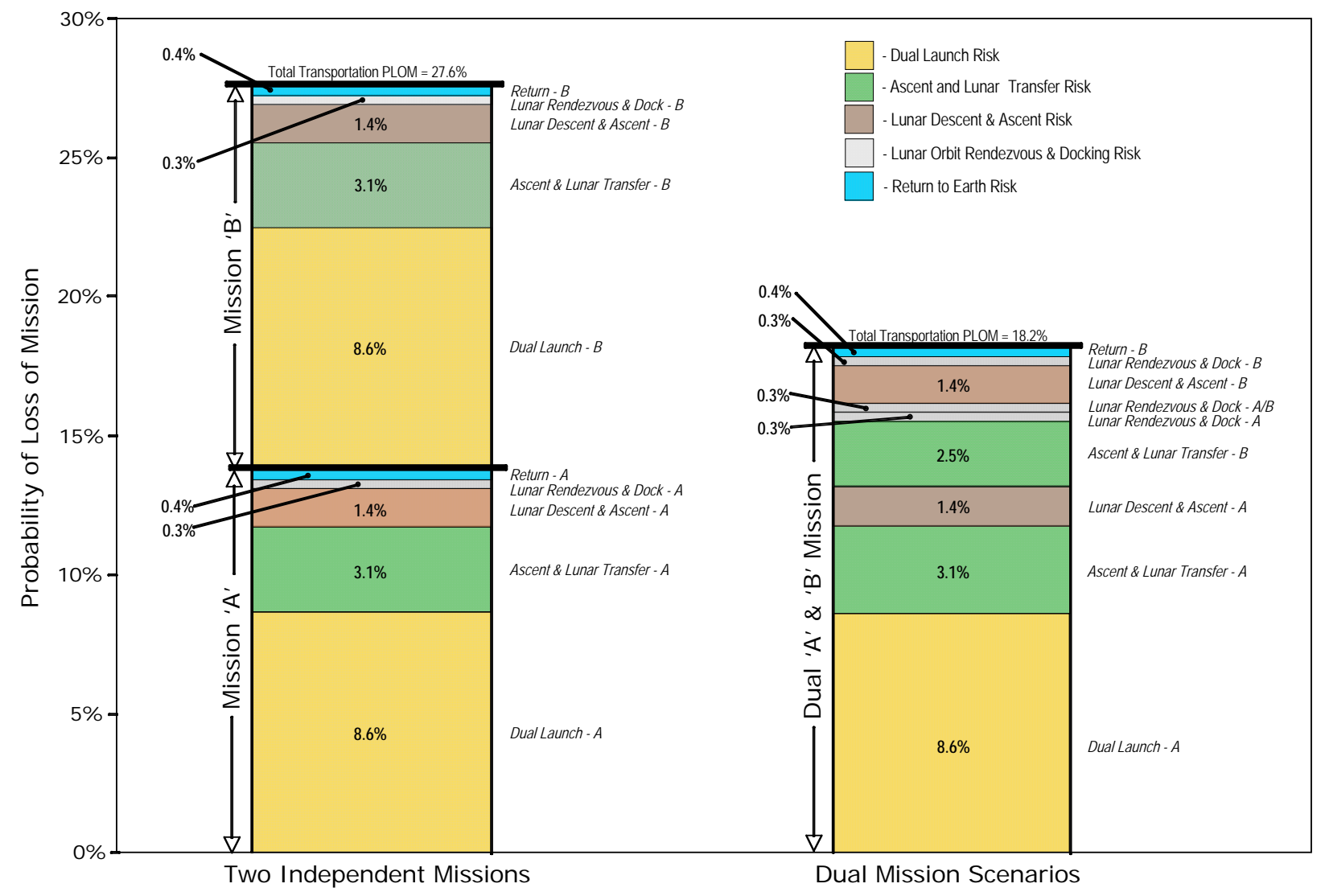

Figure 6 - Comparison of probability of loss of mission

an effective benefit of $15 \%$ relative to the Constellation Program two-mission-per-year reference.

The risk reduction in the dual mission scenario is primarily the net result of elimination and addition of mission phases. The overall PLOC is reduced by the elimination of a launch and lunar transfer phase, as well as an earth return phase, as compared with two independent missions. The elimination of these two phases reduces PLOC by $0.56 \%$. However, in the dual mission scenario, there is an additional lunar orbit rendezvous and docking event when the Mission A CEV performs a rendezvous with the Mission B Lander. This additional event increases crew PLOC by $0.26 \%$.

It is important to note that while the total probability of loss of crew over two crewed landings is decreased, the actual risk to any given crew increases in a dual mission scenario. For an independent mission, each crew is exposed to a PLOC of $0.97 \%$ for the transportation phases of the mission. In a dual mission, a single crew is exposed to the total PLOC of $1.64 \%$ in the transportation phases which is an effective increase in risk for a given crew of $69 \%$.

\section{DUAL SORTIE MISSIONS}

The dual sortie mission concept provides a means to access regions of the lunar surface that may not be accessible with an independent sortie mission due to performance limitations of the integrated transportation system. As such, this concept potentially provides expanded science and exploration capabilities beyond the mobile capabilities of the South Pole Outpost surface systems.

This mission scenario, illustrated in figure 8 , is enabled by the concept of a Mission A supersortie. A supersortie is a 30- to 90-day mission to a base which has been predeployed by an autonomous, uncrewed Cargo Lander or transferred from the South Pole Outpost via autonomous mobile systems. This supersortie site may be located anywhere on the lunar surface. Pre-deployed cargo will most likely include a surface habitat with crew provisions, power generation and storage capability, surface mobility including pressurized and unpressurized rovers, communication and navigation equipment, and science cargo. This base will be designed to be crew-tended (which is Mission A in the dual sortie scenario) and self-sustainable between periodic crew visits.

A supersortie mission of 30- to 90-days duration is necessary for Mission A in the dual sortie scenario since adequate time is needed to ensure that the Mission B Ares V is likely to launch. That is, the extended 30- to 90-day Mission A provides sufficient duration to protect for multiple launch delays due to inclement weather or hardware issues. 


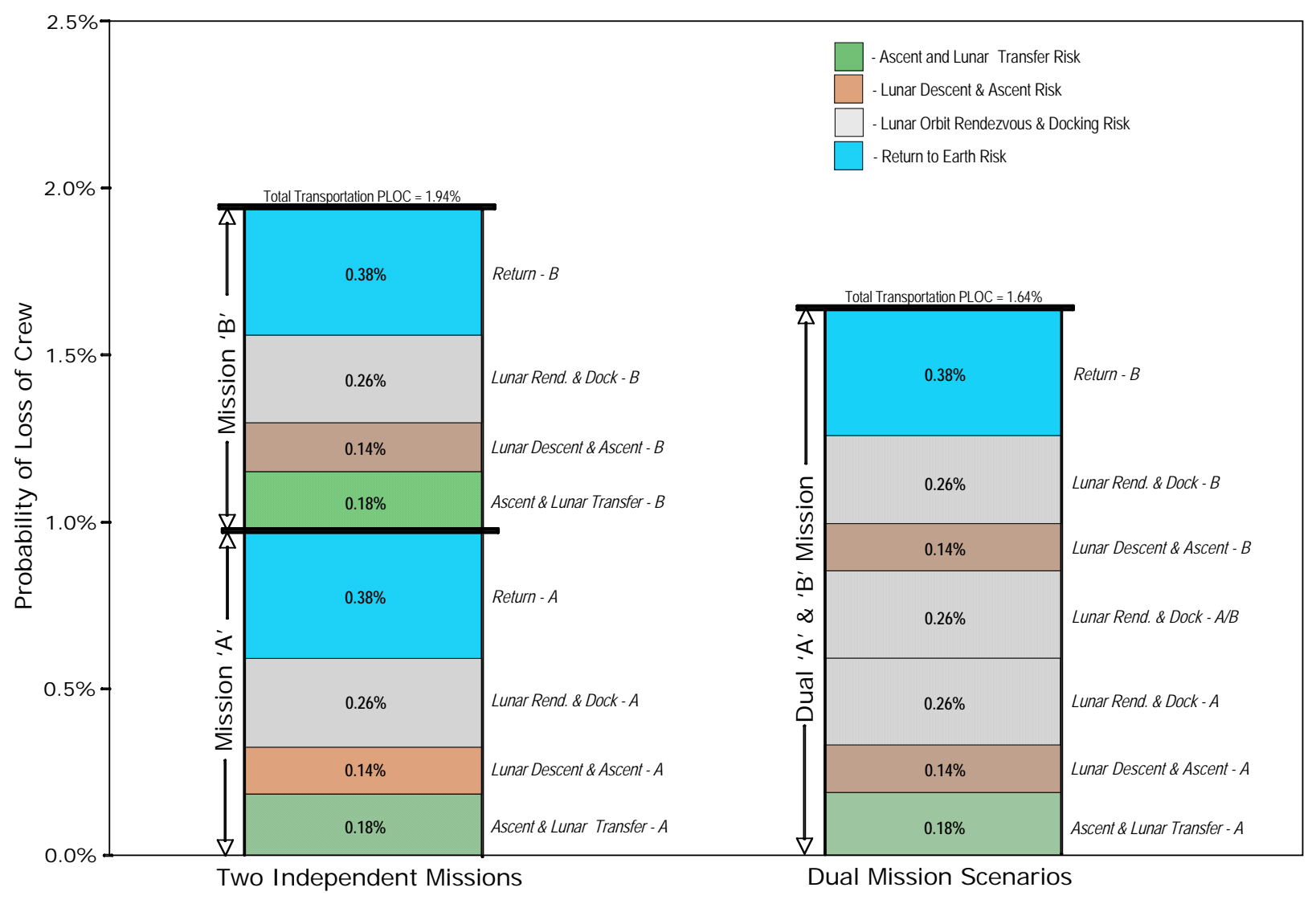

Figure 7 - Comparison of probability of loss of crew

The Mission A supersortie is conducted similar to the Constellation Program reference for a 30- to 90-day South Pole Outpost mission. Two options exist for the Mission A CEV lunar orbit. These options include a lunar polar orbit with the proper ascending node for the Mission A site or a 7-day "anytime return" orbit which minimizes the wedge angle between the Mission A landing site and the orbit for a 7 day period. These options provide differing capabilities for payload to the lunar surface, Mission A launch windows for return to the CEV, and surface accessibility for the Mission B sortie. For the purposes of this analysis, a 7-day anytime return orbit was assumed. Upon landing, the crew egresses Lander \#1 and transitions to the supersortie surface habitat. At the completion of Mission A, the crew returns to the CEV in low lunar orbit.

The Mission B sortie is initiated with the launch of EDS \#2 and Lander \#2 prior to completion of Mission A. Unlike Mission A, this mission is a direct launch to the moon since there is no requirement to rendezvous in low-Earth orbit. As in the Outpost-based sortie scenario, EDS \#2 performs a portion of LOI to utilize all remaining propellant; Lander \#2 then completes the LOI maneuver sequence and enters the CEV low lunar orbit. After the crew returns to the CEV and expends the Lander \#1 Ascent Module, the CEV performs a rendezvous and docks with Lander \#2.
Lander \#2 propulsion is utilized to maneuver the Lander \#2/CEV stack to the proper orbit plane for "anytime return" at the Mission B sortie site. The crew loiters in lunar orbit from 1 to 7 days and then descends to the Mission B site. At the completion of a 4- to 7-day sortie, the crew returns to the CEV for return to Earth.

Figure 9 illustrates the orbital geometry and associated surface access for dual sortie missions. To begin the maneuver sequence, the crew performs a coplanar ascent from the Outpost to the CEV. Similar to the Outpost-based sortie mission scenario, the orbit for the Mission B sortie site is determined by the requirement for "anytime return" to the CEV from the lunar surface and the requirement for a coplanar descent to the lunar surface. A specific orbit exists for a given Mission B landing site that minimizes the wedge angle between the landing site and the orbit over the 7 day surface stay. Complete $360^{\circ}$ longitudinal access for Mission B is possible through the use of low-lunar orbit loiter time of up to 7 days to establish the correct orbital ascending node. Accessible latitudes are a function of lander performance and minimum payload requirement to the lunar surface. Figure 9 uses color to demonstrate the maximum payload to the surface for each possible Mission B site. The color may be translated to payload mass $(\mathrm{kg})$ using the scale shown in figure 10, as both figures show data for an Orientale Basin Mission A. 


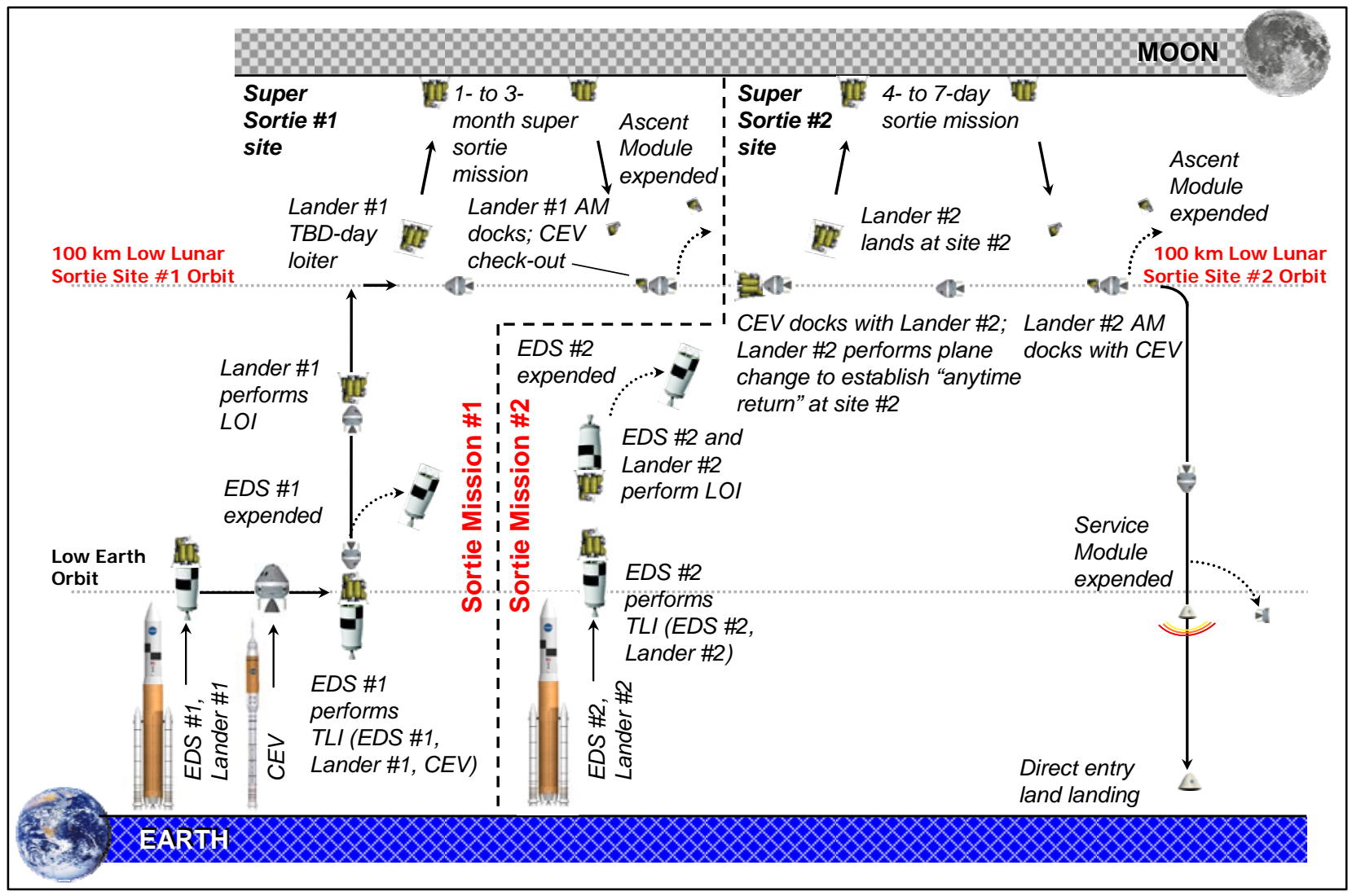

Figure 8 - Dual sortie mission scenario

\section{Performance Benefits}

Two examples are provided to demonstrate possible options for dual sortie missions: Mission A to Orientale Basin followed by Mission B to Aristarchus Plateau, and Mission A to Aristarchus Plateau followed by Mission B to Central Far Side Highlands.

As illustrated in figure 10, Lander \#1 delivers over $5 \mathrm{t}$ of payload with a crew of 2 to the Orientale Basin site with 24 hours of crew support logistics. For the Mission B sortie to Aristarchus Plateau, Lander \#2 delivers $4.8 \mathrm{t}$ of payload with 2 crew and resources for a 7 day surface stay. Additional LEAG-defined priority sites for Mission B are reachable from Orientale Basin including Rim Bode and Mare Tranquillitatis.

Figure 11 illustrates the Mission B accessible region from a Mission A supersortie to Aristarchus Plateau. Lander \#1 delivers 2 crew and $2.8 \mathrm{t}$ of payload to the Aristarchus Plateau site with 24 hours of crew support logistics. Lander \#2 delivers 2 crew and 7.9 t of payload for a 7 day surface stay to Central Far Side Highlands. Other LEAG-defined priority sites are accessible from Aristarchus Plateau including Orientale Basin.

\section{Cost Savings}

The primary cost benefit of the dual sortie mission scenario is the ability to accomplish the Mission B sortie with a single launch since no Ares I launch is required. As in the Outpost-based sortie scenario, cost benefits may be estimated by comparing flight rate dependent variable/marginal costs of the dual sortie mission scenario with the Constellation Program reference two-mission-peryear campaign.

Figure 5 illustrates that the Outpost-based sortie scenario can lead to an annual variable savings of $\sim 8 \%$ versus the Constellation Program reference two-mission-per-year campaign. These savings are related to the variable contribution of one less Ares I/CEV flight in context of the variable costs of Ares I, Ares V, CEV, Lander, and Ground Operations. This estimate is based on the author's understanding of current Constellation Program estimates including certain assumptions considering limited data sets.

\section{Risk Reduction}

Dual sortie mission scenarios potentially offer a reduction in the overall PLOM and in the overall PLOC compared with the Constellation Program reference two-mission-per-year lunar campaign. 


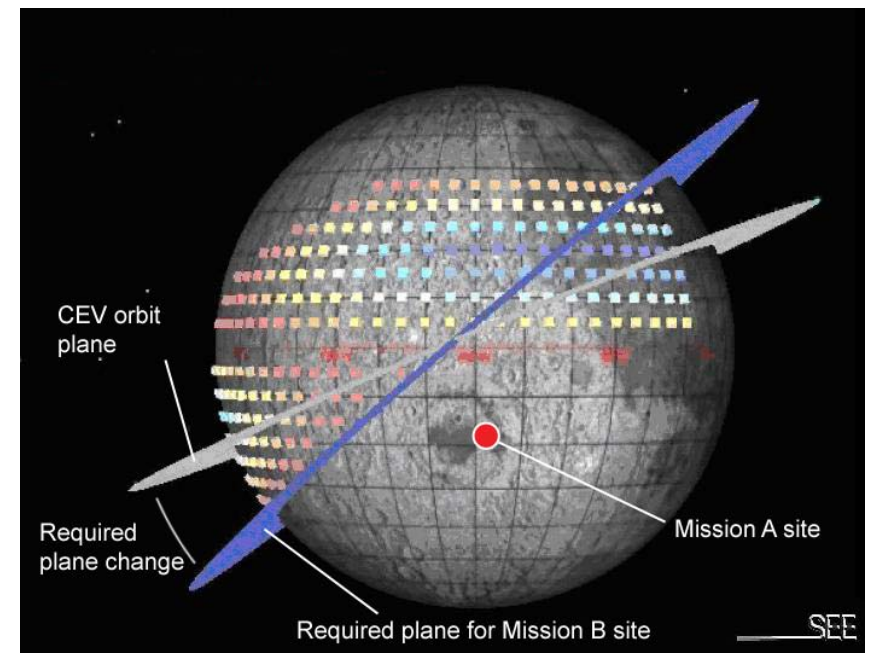

Figure 9 - Plane change capability enabling dual sortie missions

As described above, the evaluation of PLOM and PLOC risks was limited to the mission phases that are associated with the integrated transportation system. The five mission phases that were evaluated include: dual launch, ascent and lunar transfer, lunar descent and ascent, lunar rendezvous and docking, and return to Earth. Additionally, all risks in this analysis were evaluated assuming transportation system maturity.

Figure 6 shows that dual mission scenarios can provide a significant risk reduction in PLOM. The total PLOM for the transportation phases across two baseline crewed missions to the lunar surface is $27.6 \%$. A dual mission scenario reduces the overall PLOM for two crewed landings to $18.2 \%$ resulting in an effective $34 \%$ reduction in the integrated transportation system PLOM components relative to the Constellation Program two-mission-per-year reference.

Figure 7 shows a comparison of the integrated transportation system PLOC components for the dual mission scenarios and the Constellation Program reference two-mission-per-year campaign. For two reference lunar missions, the total PLOC for the transportation phases is $1.94 \%$. The Outpost-based sortie scenarios reduce the integrated transportation system PLOM components for two crewed landings to $1.64 \%$ resulting in an effective benefit of $15 \%$ relative to the Constellation Program two-missionper-year reference.

As with all dual mission scenarios, the total probability of loss of crew over two crewed landings is decreased while the actual risk to any given crew increases. For an independent mission, each crew is exposed to a PLOC of $0.97 \%$ for the transportation phases of the mission. In a dual mission, a single crew is exposed to the total PLOC of $1.64 \%$ which is an effective increase in risk for a given crew of $69 \%$.

\section{ENHANCED OUTPOST DEPLOYMENT}

The enhanced Outpost deployment scenario provides a means for delivering greater payload mass to the lunar surface than with an independent crewed lander mission and provides a fully redundant, backup Lander Ascent Module on the lunar surface.

Figure 12 illustrates the dual mission enhanced Outpost deployment scenario. Mission A is similar to the Constellation Program reference mission operations concept for Outpost deployment. After descent to the South Pole Outpost site, the crew deploys Outpost infrastructure during a Mission A surface stay of up to 3 months. Following completion of Mission A, the crew remains at the Outpost to conduct Mission B.

Mission B begins with an Ares V launch of EDS \#2 and Lander \#2. EDS \#2 performs TLI. To optimize propellant usage, EDS \#2 performs a portion of LOI to utilize the remainder of its propellant load; Lander \#2 then completes the LOI maneuver sequence. After a lunar orbit loiter, Lander \#2 lands autonomously at the lunar Outpost. The crew then deploys Outpost infrastructure during a Mission B surface stay of up to 3 months. At the completion of Mission B, the crew returns to the CEV with the Lander \#1 Ascent Module and returns to Earth.

\section{Performance Benefits}

Payload-to-the-lunar-surface capability is substantially increased in Mission B as a result of the additional propellant available for Lander \#2 descent. In this scenario Lander \#2 is able to deliver $13.8 \mathrm{t}$ to the South Pole Outpost vs. Lander \#1 capability of $6 \mathrm{t}$. This performance is potentially sufficient to deliver any single Outpost element including an outfitted habitat segment for a multi-segment surface habitat configuration.

\section{Cost Savings}

The primary cost benefit of the enhanced Outpost deployment mission scenario is the ability to accomplish Mission B with a single launch since no Ares I launch is required. As in the Outpost-based sortie scenario and the dual sortie scenario, cost benefits may be estimated by comparing flight rate dependent variable/marginal costs of the dual sortie mission scenario with the Constellation Program reference two-mission-per-year campaign.

Figure 5 illustrates that the enhanced Outpost deployment scenario can lead to an annual variable savings of $\sim 8 \%$ versus the Constellation Program reference two-missionper-year campaign.

\section{Risk Reduction}

A risk reduction benefit is realized in the enhanced Outpost deployment scenario since a spare Lander will be available 


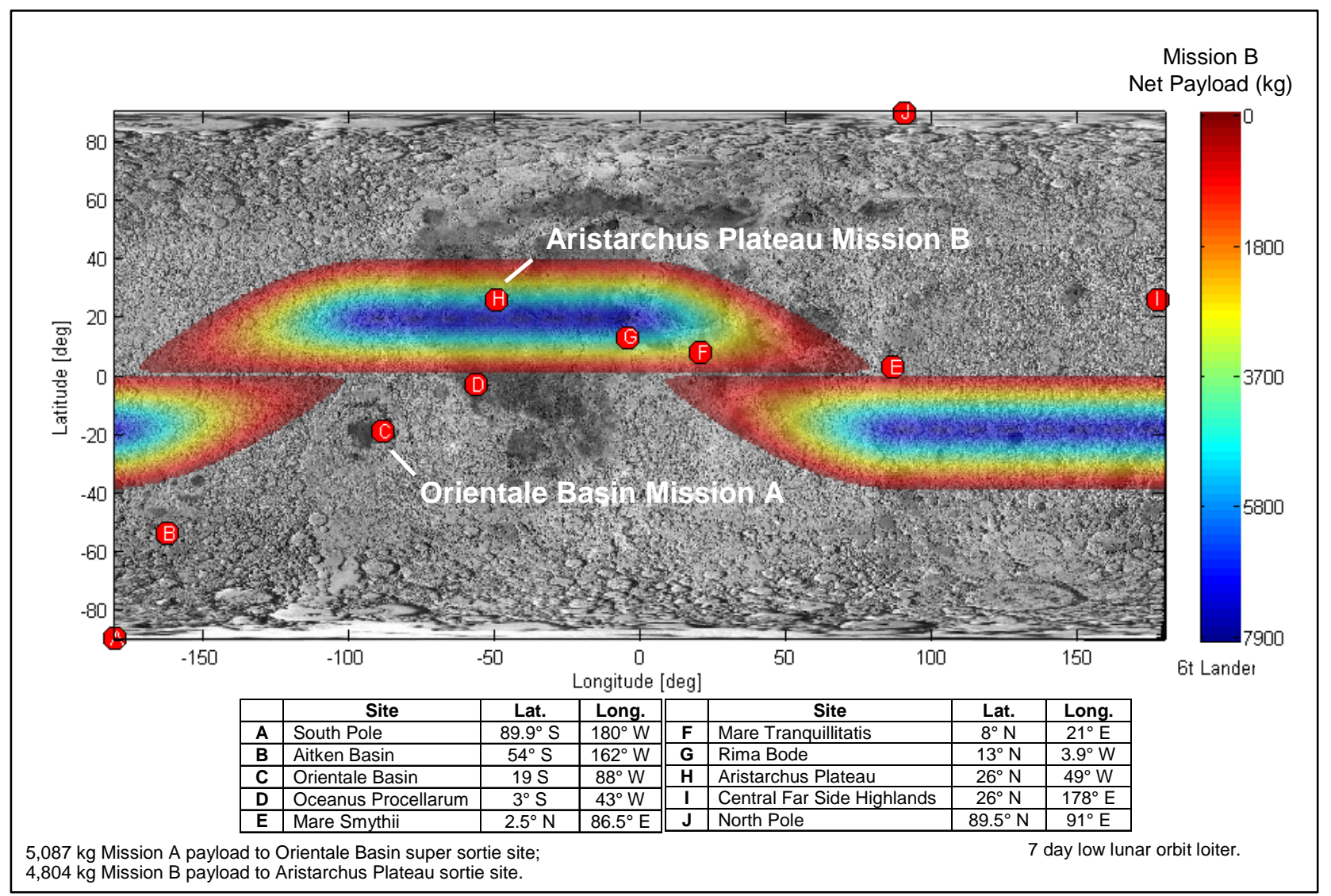

Figure 10 - Example dual sortie from Orientale Basin to Aristarchus Plateau

at the Outpost throughout the duration of Mission B and for all future missions. This provides a fully redundant, backup Lander Ascent Module if the primary Ascent Module fails during any future mission.

The probability of an Ascent Module failure on any mission that would require the use of the spare Ascent Module for return to the CEV is highly dependent on surface stay time. For example, PLOM due to Ascent Module failure at 30 days is $0.65 \%$; PLOM due to Ascent Module failure at 180 days is $1.87 \%$. PLOC due to Ascent Module failure at 30 days is $0.15 \%$; PLOC due to Ascent Module failure at 180 days is $0.43 \%$. By inherently providing a backup Ascent Module capability, this dual mission scenario reduces these probabilities which are a major contributor to lunar surface operational risk. Additionally, if this dual mission scenario is used multiple times for Outpost deployment, the availability of additional Landers at the Outpost provides a means for Lander component sparing and scavenging.

\section{AdvanCEd CAPABILITY OPTIONS}

Additional mission scenarios are potentially possible with the development of advanced capabilities. These include abort to the lunar surface options for lunar ascent/descent and Lander reusability options.

\section{Expanded Lunar Abort Modes}

Return-to-Outpost abort modes for lunar ascent and/or descent are potentially feasible with the incorporation of Lunar Lander contingency landing capability. These additional abort modes exist since a redundant, backup Lander Ascent Module is based at the Outpost with the enhanced Outpost deployment scenario and may be utilized for crew ascent in the event of a contingency. Descent abort modes may potentially be expanded to include a "Press to Outpost" capability. Enabled ascent abort modes may include an "Abort Once Around" capability (AOA, analogous to the Space Shuttle AOA abort mode) in the event that the Lander Ascent Module can achieve only a degraded orbit. Advanced capabilities necessary for these aborts include Lander Ascent Module structural capability to survive a contingency landing ("legs" or other structural enhancements), capability for the Ascent Module main engine and/or reaction control system to throttle for landing, and guidance and navigation capability to return to the Outpost and execute a landing.

\section{Lander Reusability Options}

Additional cost benefits of the dual mission scenarios may potentially be realized with the development of a partially reusable Lunar Lander. Specifically, in these dual mission scenarios, the capability to refuel and refurbish the Lander \#1 Ascent Module in lunar orbit may leverage the cost of a 


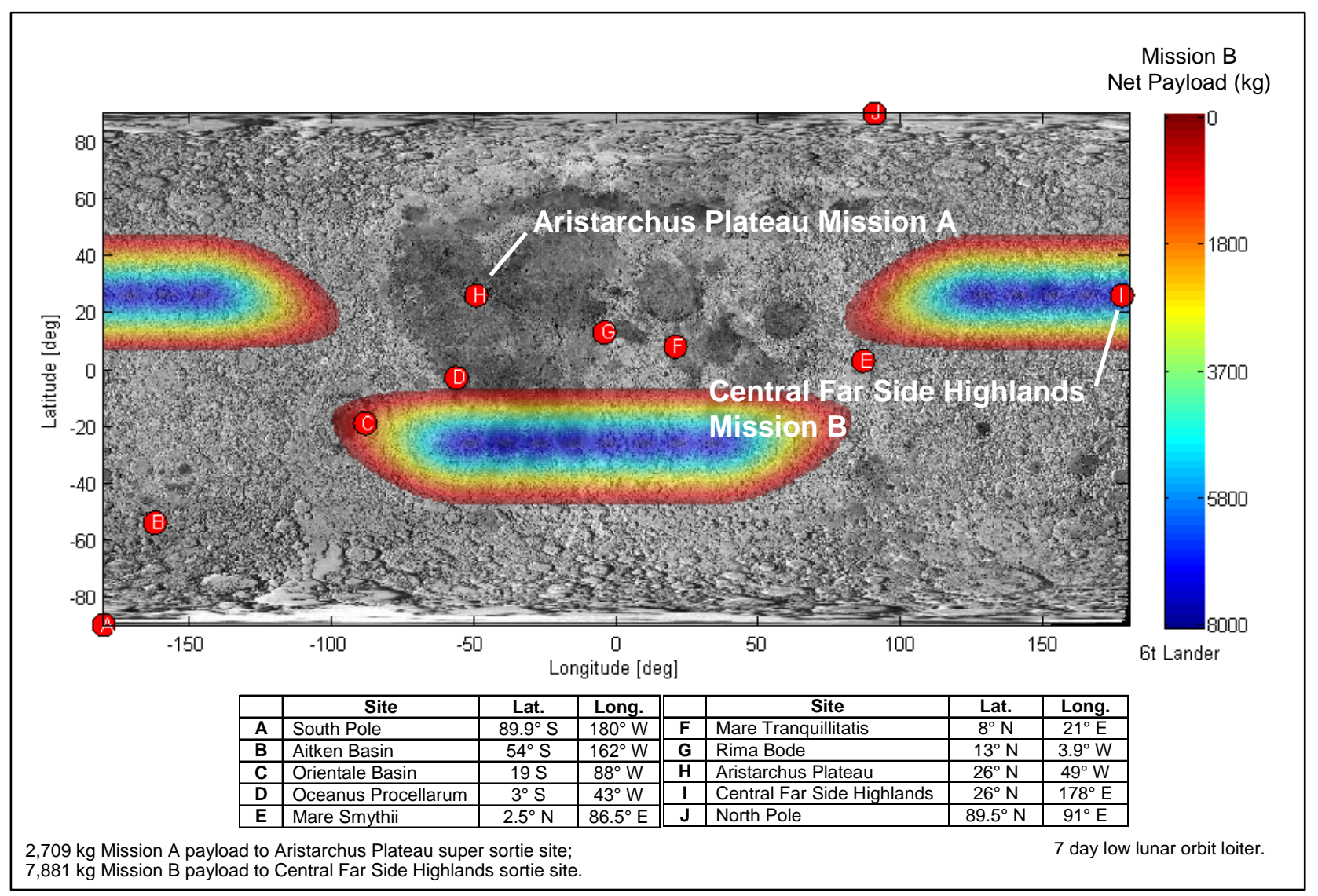

\section{Figure 11 - Example dual sortie from Aristarchus Plateau to Central Far Side Highlands}

Lander across two missions. In this scenario, a new Lander Descent Module and refueling capability for the Ascent Module are launched on an Ares $\mathrm{V}$ and delivered to the CEV/Lander \#1 Ascent Module stack in lunar orbit. Options for refueling include propellant transfer (existing technology for hypergolic propellants) and use of propellant "kits" for the exchange and replacement of propellant tanks.

\section{CONCLUSiOnS}

\section{Summary}

The Outpost-based sortie mission scenario is an attractive means to reach higher latitude LEAG-defined priority lunar sites considering performance, cost and risk. The Mission B sortie may be performed at the completion of an Outpost buildup flight or Outpost utilization flight. A Mission B sortie to the North Pole is a unique option since payload capability is maximized at this location. This payload capability may provide the opportunity to establish a miniOutpost at the North Pole for enhanced science, commercial activities, or in support of an international partner.

The dual sortie mission scenario is enabled by the establishment of supersortie infrastructure at one or more locations on the lunar surface. Supersortie infrastructure may be delivered via an autonomous cargo lander or may be transported by mobile systems from the South Pole Outpost. The ability to access substantial regions of the lunar surface from a given supersortie location can enable exploration of many sites of high scientific interest as defined by the LEAG. Mission combinations which are possible include a supersortie Mission A followed by a sortie or supersortie Mission B.

The enhanced Outpost deployment scenario offers potential solutions - within current technology and operations planning - to significant issues under study by the Constellation Program. Specifically, this scenario addresses performance issues by providing a robust capability to deliver high mass cargo to the lunar surface on a Lander configured for crew transport. This scenario addresses a significant surface operations risk by inherently providing a fully redundant, backup Lander Ascent Module which may be used if the primary Ascent Module fails to launch. Additionally, the enhanced Outpost deployment scenario provides a means for Lander component sparing and scavenging on the lunar surface.

These three dual mission scenarios also provide a public relations benefit since they are substantially different than Apollo and provide a relatively cost effective means for lunar exploration across multiple sites of interest while maintaining a permanent South Pole Outpost.

\section{Future Work}

In the dual mission scenarios, the EDS is required to operate in the lunar vicinity to perform part of the LOI maneuver 


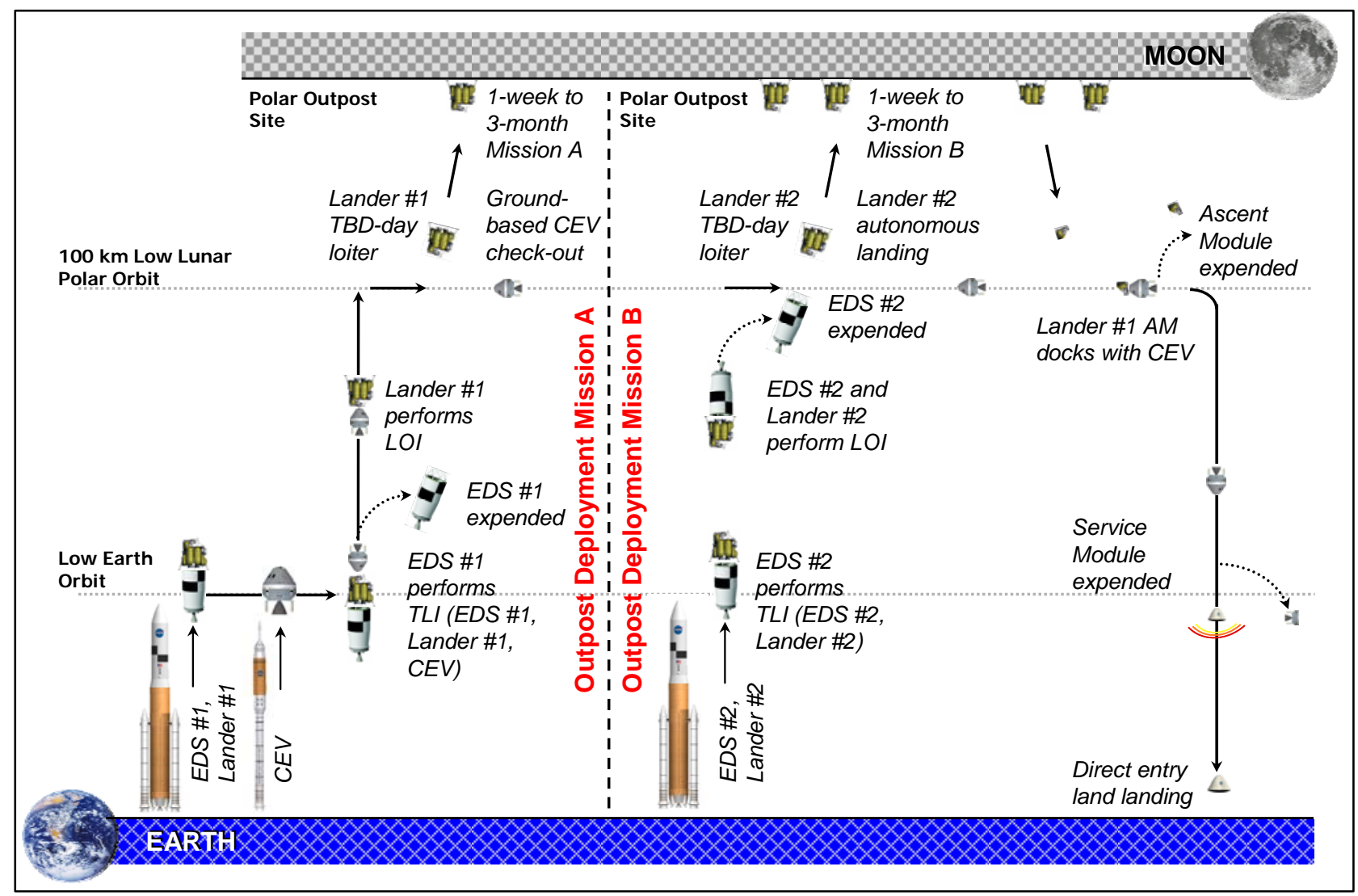

Figure 12 - Enhanced Outpost deployment mission scenario

sequence. To accomplish this, the EDS will require long range communication and navigation capability. Additionally, the EDS J2-X main engine may require prefire conditioning prior to LOI. Further study is needed to assess these potential EDS requirements.

The LOI maneuver sequence is split between the EDS and Lander in order to maximize Mission B payload mass to the lunar surface. For this current assessment, operational considerations for this split maneuver sequence were not addressed. Analysis is needed to determine how to optimally perform this split maneuver sequence considering guidance, navigation, and control issues.

After the EDS LOI maneuver sequence, the EDS will perform a disposal burn. Future study is needed to determine the optimal disposal method. Options include disposal via a lunar impact trajectory or a heliocentric trajectory.

These dual mission scenarios require a CEV rendezvous and docking in lunar orbit to enable crew transfer to Lander \#2 prior to the Mission B descent to the lunar surface. Future analysis is needed to evaluate the CEV Service Module propellant budget including this additional rendezvous. If the CEV Service Module can not accommodate this additional rendezvous, Lander \#2 may potentially serve as the active, chaser vehicle for rendezvous with the CEV.

\section{REFERENCES}

[1] Constellation Architecture Requirements Document (CARD), CxP 70000, Release A, August 13, 2007.

[2] Phase Two Summary Report (Draft), NASA Lunar Architecture Team, October 9, 2007.

[3] Michelle A. Garn, "Joint APO/LSAM Office Analysis on Nominal and Contingency Delta-V Requirements, LOI Delta-V Refinement Final,” APO-00-1005, June 28, 2007.

[4] Lunar Exploration Analysis Group Web Site http://www.lpi.usra.edu/leag/

\section{BIOGRAPHIES}

Rudy Saucillo currently serves as a Systems Analysis Lead in the Space Mission Analysis Branch at the NASA Langley Research Center. In this role, Mr. Saucillo initiates and leads Center and multi-Center systems studies of human and robotic space exploration architectures including transportation vehicle and surface element conceptual designs and operations concepts. Previously, he was a technical lead and engineering manager with the Boeing Company. His responsibilities included directing a NASA 
Langley technical support contract responsible for the systems engineering and analysis of advanced space and atmospheric systems. He has a Bachelor of Science degree in Aerospace Engineering from Texas A\&M University and a Master of Science degree in Aerospace Engineering from the University of Michigan.

David Reeves is an aerospace engineer in the Space Mission Analysis Branch at the NASA Langley Research Center. His specialties include preliminary vehicle design and sizing, as well as architecture development and integration, with the majority of his work focusing on human lunar landers and lunar exploration architectures. He has previously worked for Analytical Mechanics Associates and the National Institute of Aerospace. He holds a Bachelor of Science degree in Engineering Physics from the University of Oklahoma and a Master of Science degree in Aerospace Engineering from the Georgia Institute of Technology.

Jonathan Chrone is a staff engineer with Analytical Mechanics Associates of Hampton, VA. He has been an onsite contractor at the NASA Langley Research Center since 2005 where he has been involved with various lunar exploration projects including trade studies detailing the requirements on lunar orbit insertion to allow for full lunar surface access, and the effects of the Earth-Moon geometric cycle on the frequency and length of translunar injection windows. He has also been part of the development team for the Synergistic Engineering Environment, an engineering visualization and analysis tool developed at Langley Research Center. He graduated from Virginia Tech in 2005 with a Bachelor of Science degree in Aerospace Engineering.

David North is an aerospace engineer in the Space Mission Analysis Branch at the NASA Langley Research Center and has provided mission analysis and conceptual design engineering for NASA's space exploration program since 2004. He is currently serving as the mechanical engineering lead for the Constellation Program Lunar Lander Project Office. He has held management positions at Allied Aerospace in 2003 and Siemens Westinghouse Corporation in 2001. From 1988 to 2000, he was a mechanical engineer for Pratt \& Whitney in the Advanced Engineering Group. He has a Bachelor of Science degree in Aerospace Engineering from the University of Virginia and a Master of Science degree in Aerospace Engineering from the University of Texas. 


\section{Dual Mission Scenarios for the Human Lunar Campaign - Performance, Cost, and Risk Benefits}

2008 IEEE Aerospace Conference

March 1 - 8, 2008

Big Sky, Montana 


\section{Overview}

- Scenarios for human lunar operations with capabilities beyond current planning are potentially feasible based on the concept of dual, sequential missions utilizing a common crew and a single Ares I/CEV

- New missions within baseline technology planning are potentially feasible

- Outpost-based sortie missions

- Accessibility includes higher latitude regions of high scientific interest due to concentrated solar wind volatiles

- Dual sortie missions

- Substantial surface accessibility for the Mission B sortie is defined by the Mission A site and minimum payload desired to the Mission B site

- Accessibility includes numerous combinations of priority science sites

- Enhanced Outpost deployment scenarios

- Provides substantial cargo delivery capability and addresses significant operational risk

- Primary cost benefit is that Mission B of the dual mission sequence is a "single launch solution" without the requirement to human-rate the Ares V 


\section{Discussion Topics}

- Introduction and Ground Rules \& Assumptions

- Outpost-based Sortie Missions

- Dual Sortie Missions

- Enhanced Outpost Deployment

- Conclusions 


\section{Introduction}

- The NASA Constellation Program is currently defining a lunar architecture and associated capabilities to establish a lunar South Pole Outpost beginning in 2019

- The recently completed study by the NASA Lunar Architecture Team (LAT) showed that requirements for global sortie missions as opposed to South Pole Outpost deployment and support - are the drivers for architecture performance

- The dual missions scenarios described in this paper were developed as part of the LAT study as a potential means for optimizing architecture performance 


\section{Ground Rules \& Assumptions}

The lunar lander concept used in this analysis is based on the following:

- Lander Design Analysis Cycle \#1 (LDAC \#1) configuration provided by the Constellation Program Lunar Lander Project Office. This is a conceptual, early-design-cycle lander and does not represent a Lunar Lander Project Office final design.

- Lander ascent module with $8.2 \mathrm{~m}^{3}$ total internal volume and $6 \mathrm{~m}^{3}$ habitable volume

- 3 x $15 \mathrm{klb}$ thrust LOX/H2 lander descent module engines

- $889 \mathrm{~m} / \mathrm{s}$ lunar orbit insertion (LOI) DV for the South Pole outpost

- 2,100 m/s lunar descent DV for all sites

- Up to seven-day low-lunar orbit loiter capability

- Lander sized to deliver 6 metric tons ( $\mathrm{t}$ ) of cargo to the South Pole outpost with four crew and 24 hours of crew life support logistics

- 20\% growth margin and 20\% program manager's reserve (PMR) added to lander dry mass 


\section{Ground Rules \& Assumptions \\ Lunar Lander with 2-Crew Sortie Kit}

\section{Crew Configuration}

Thermal

Radiator

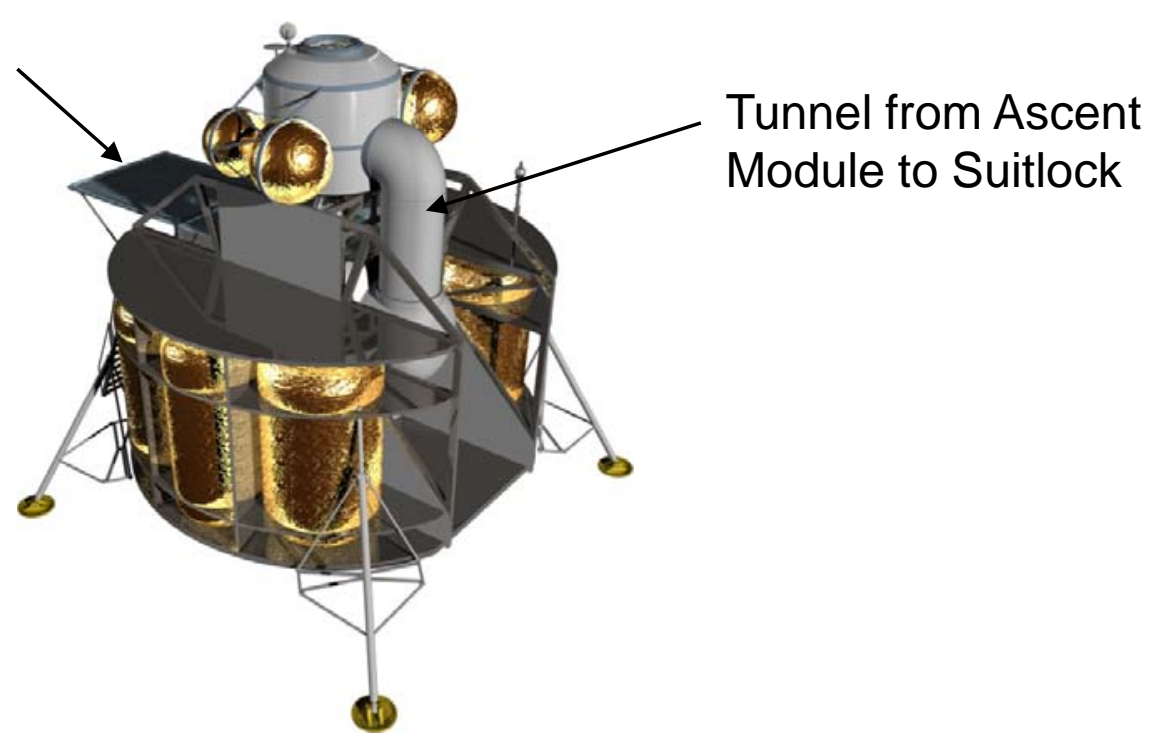

- Lander kit is designed for global access

- Up to 7 day surface stay

- Anytime return capability is preserved

- Residual descent module LOX/H $\mathrm{H}_{2}$ is scavenged for 7 day fuel cell operations

- Net payload to the surface is a function of sortie site, LLO loiter duration and surface stay time

- Cargo includes pressurized and unpressurized logistics, science payload

- 100 kg science return cargo capability

- Daytime only operations are assumed

- Launch mass of lander with kit is $56 \mathrm{t}$
Lander Kit MEL

\begin{tabular}{|r|c|}
\hline Structures & 86 \\
\hline Protection & 0 \\
\hline Power & 229 \\
\hline Thermal & 235 \\
\hline Avionics & 0 \\
\hline Life Support & 92 \\
\hline Suit-Lock Sys & 463 \\
\hline Outfitting & 0 \\
\hline 20\% Growth & 221 \\
\hline 20\% PMR & 265 \\
\hline ECLSS Consumables & 105 \\
\hline Power Consumables & 0 \\
\hline Total & $\mathbf{1 , 6 9 6}$ \\
\hline
\end{tabular}




\section{Outpost-Based Sortie Missions}




\section{Outpost-based Sortie Mission Scenario}

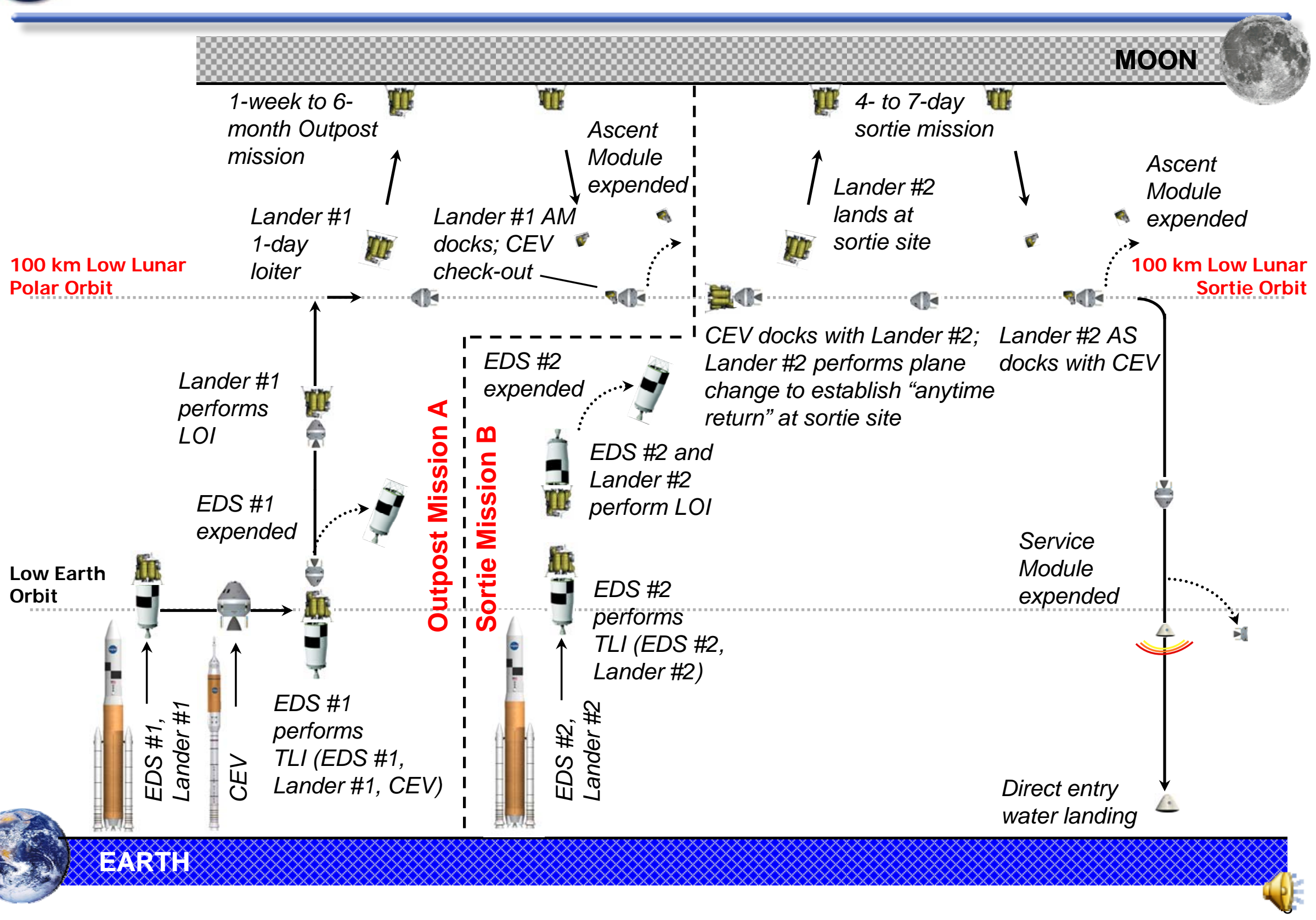




\section{Orbital Plane Change Capability Enabling Outpost-Based Sorties}

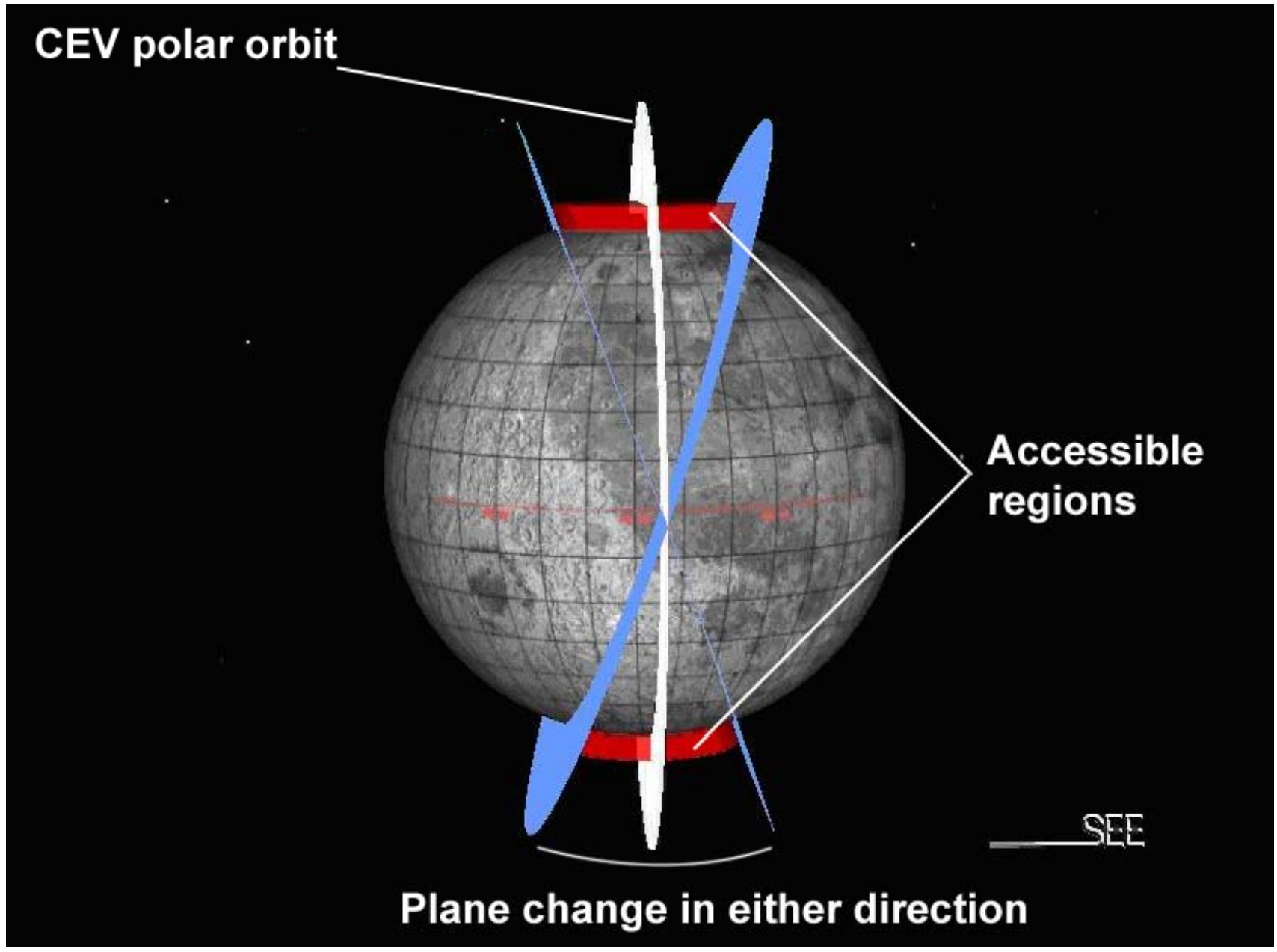




\section{6t Capability to the South Pole Outpost Surface Access for Outpost-based Sortie Mission}
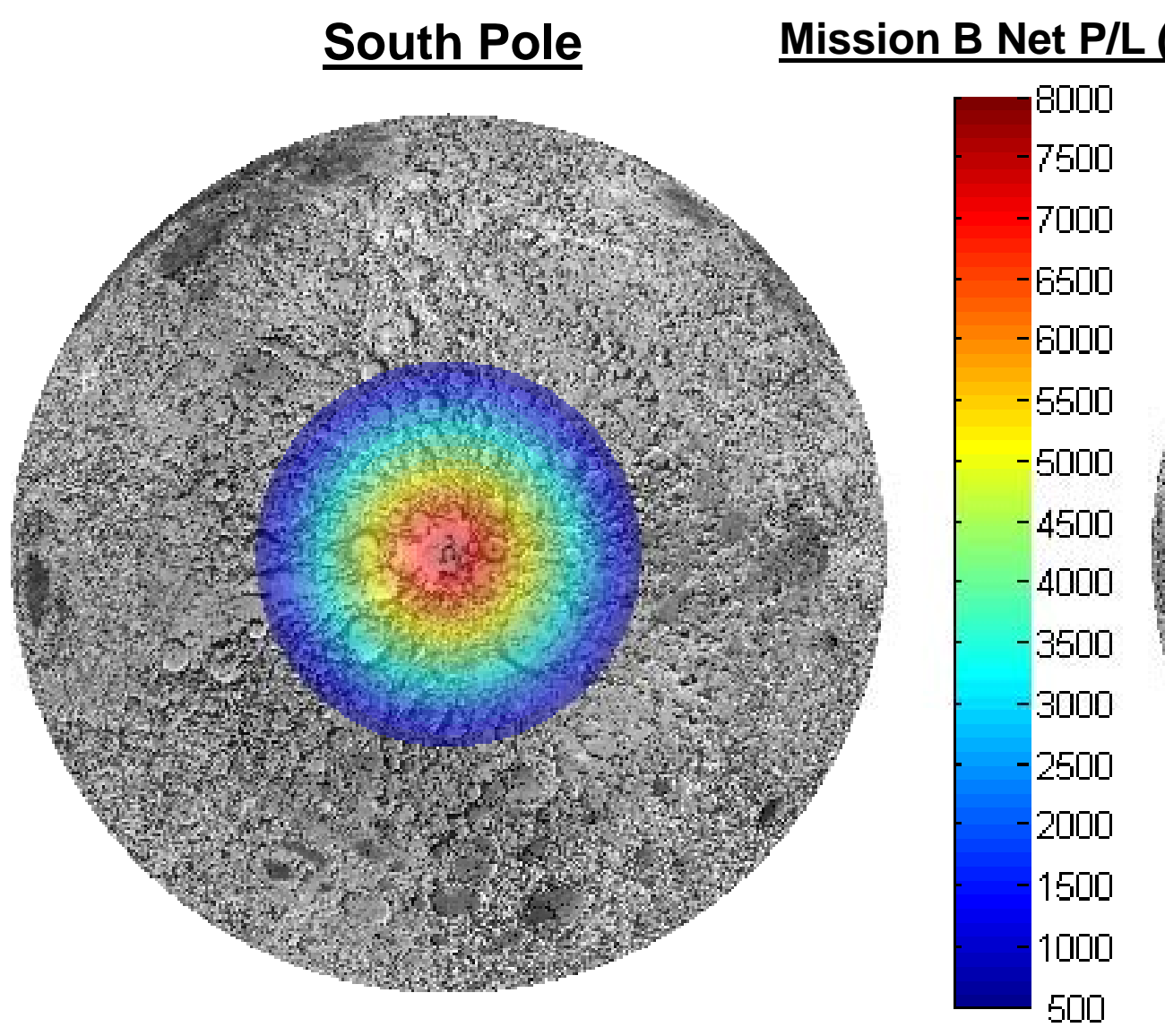

(kg) North Pole

Lander Assumptions:

- 2 crew

- 7 day stay

- $889 \mathrm{~m} / \mathrm{s}$ LOI capability to South Pole Outpost

\begin{tabular}{|c|c|c|c|}
\hline $\begin{array}{c}\text { Payload to } \\
\text { the Surface } \\
(\mathrm{kg})\end{array}$ & $\begin{array}{c}\text { Latitude } \\
\text { (deg.) }\end{array}$ & $\begin{array}{c}\text { LSAM \#2 + } \\
\text { CEV Plane } \\
\text { Change } \\
\text { (deg.) }\end{array}$ & $\begin{array}{c}\text { Surface } \\
\text { Access } \\
(\%)\end{array}$ \\
\hline 7,000 & \pm 86.8 & 2.63 & 0.15 \\
\hline 5,000 & \pm 80.9 & 7.53 & 1.26 \\
\hline 3,000 & \pm 74.4 & 12.79 & 3.69 \\
\hline 1,000 & \pm 67.9 & 18.45 & 7.35 \\
\hline
\end{tabular}




\section{Comparison of Total Variable Costs*}

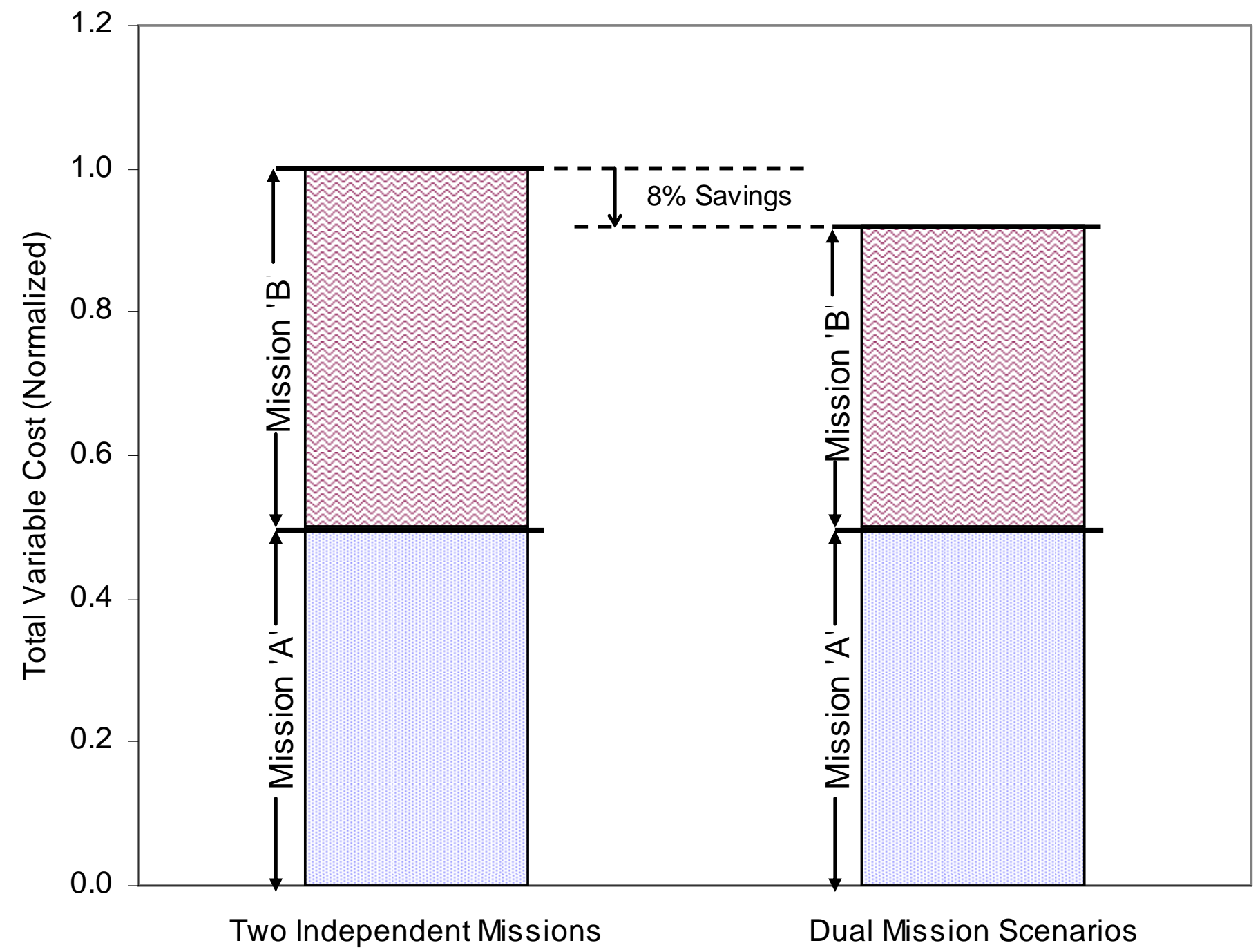

* Fixed costs not included. 


\section{Comparison of Probability of Loss of Mission}

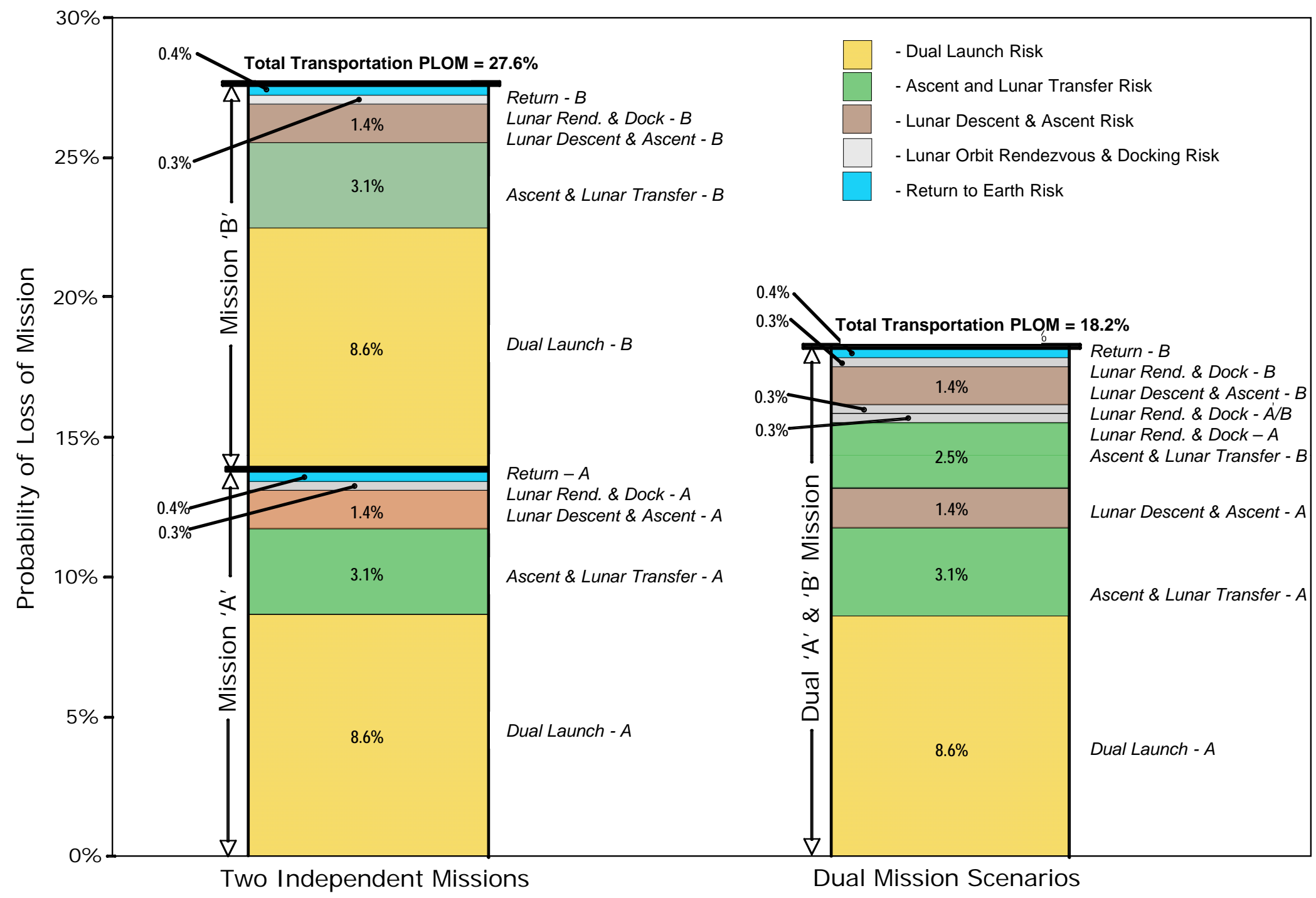




\section{Dual Sortie Missions}




\section{Dual Sortie Mission Scenario}

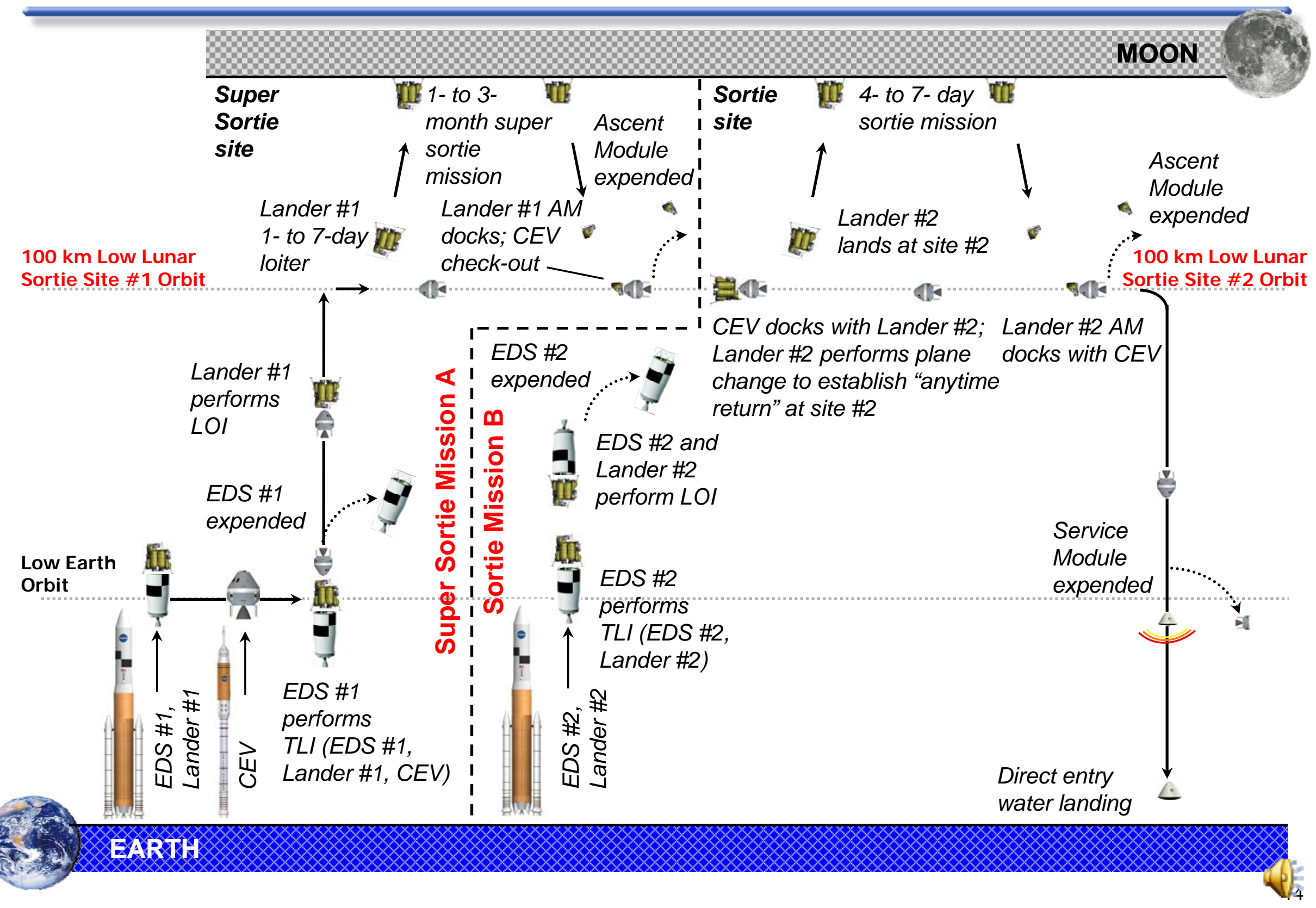




\section{Crew, 7 Day Loiter, 7 Day Stay}

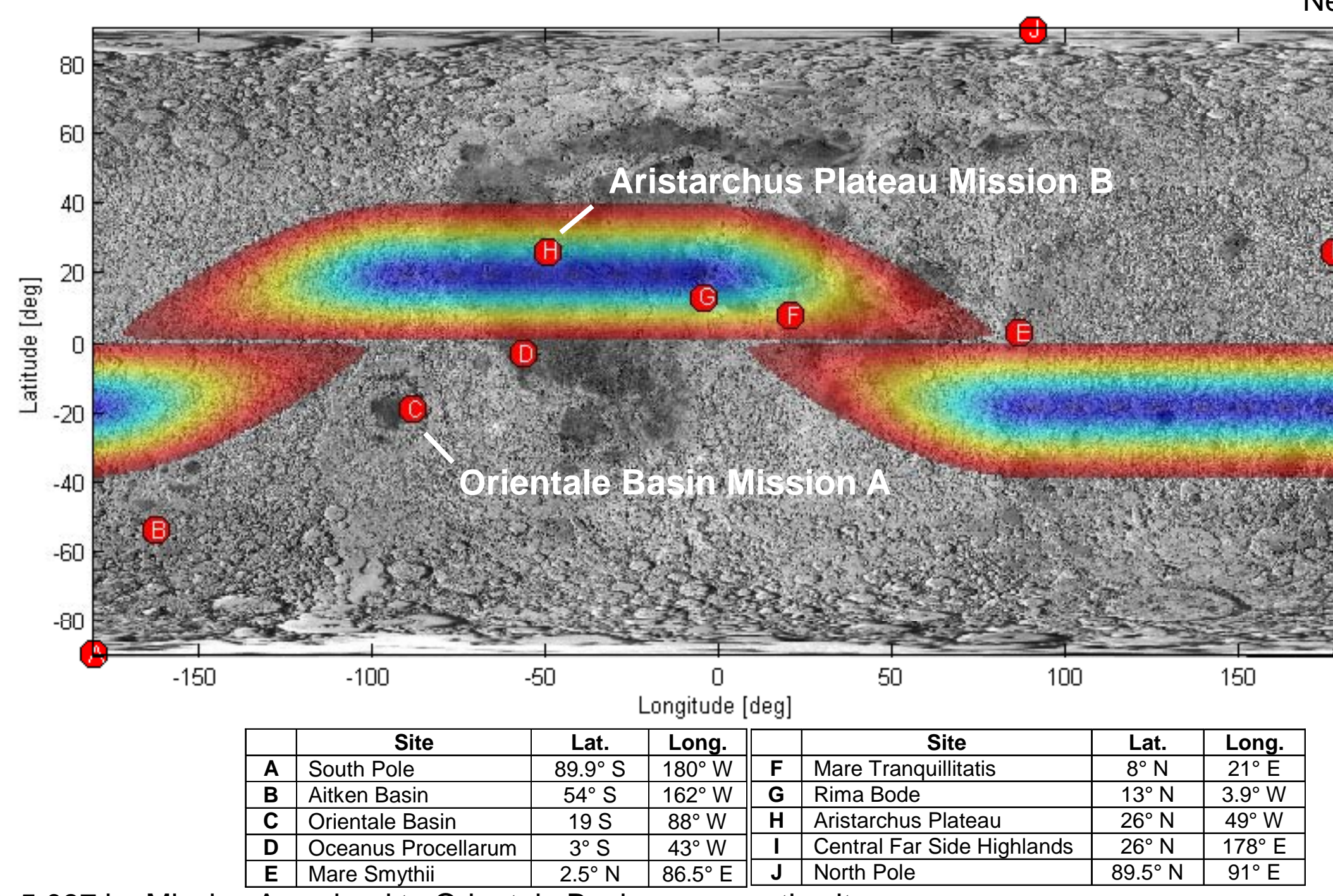

Mission B Net P/L (kg)

* $5,087 \mathrm{~kg}$ Mission A payload to Orientale Basin super sortie site; 4,804 kg Mission B payload to Aristarchus Plateau sortie site.

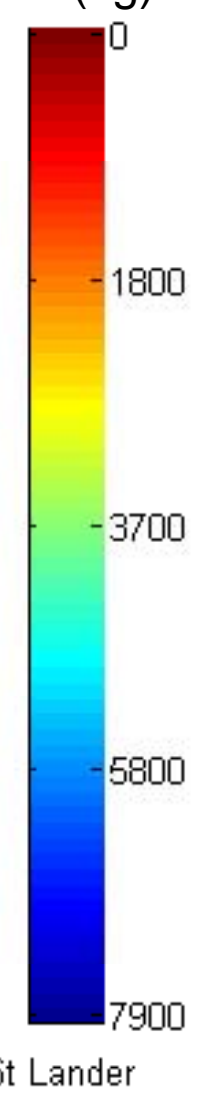

6t Lander 


\section{Enhanced Outpost Deployment}




\section{Enhanced Outpost Deployment Mission Scenario}

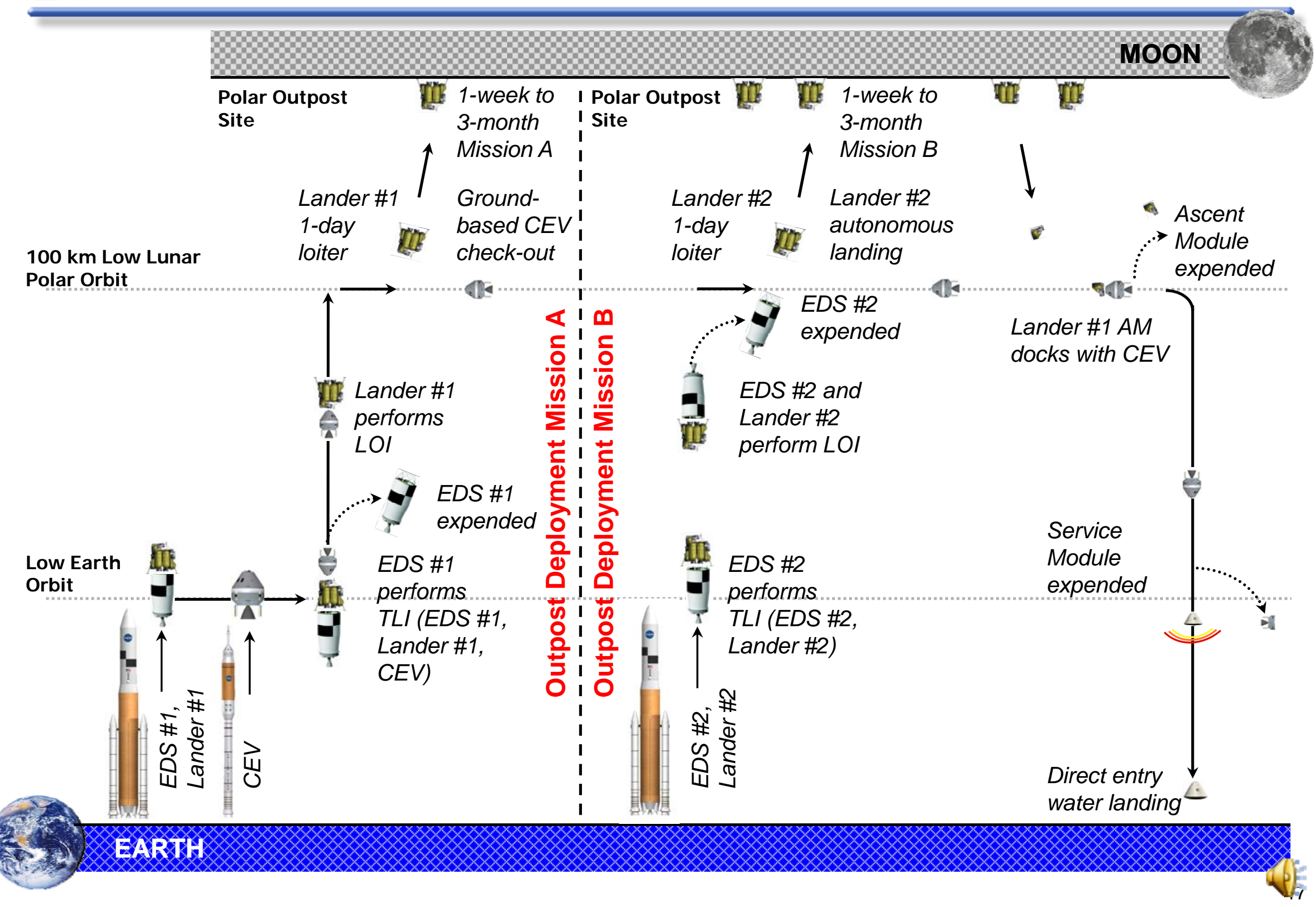




\section{Conclusions and Future Work}

- The Outpost-based sortie mission scenario is an attractive means to reach higher latitude priority lunar sites considering performance, cost, and risk

- The dual sortie mission scenario provides the capability to access substantial regions of the lunar surface from a given supersortie location enabling exploration of many sites of high scientific interest

- The enhanced outpost deployment scenario offers potential solutions to significant issues under study by the Constellation Program

- Ability to deliver high mass cargo on a lander configured to deliver crew

- Substantially reduces mission risk by providing a fully redundant, spare ascent module

- Provides an inherent means for the sparing and scavenging of lander components

- Future work:

- Incorporate dual scenarios in Constellation Program integrated transportation system analyses assessing performance, cost, and risk 


\section{Authors}

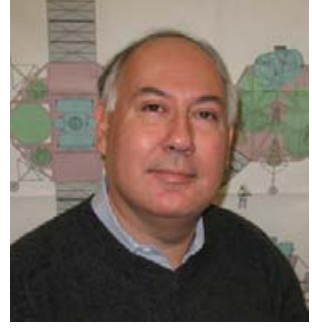

Rudy Saucillo NASA LaRC

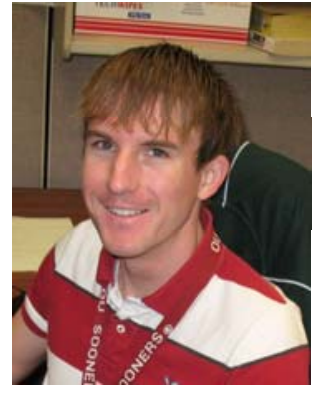

David Reeves NASA LaRC

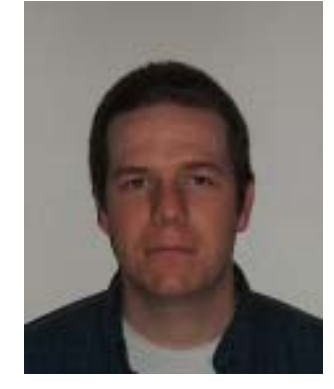

Jon Chrone AMA, Inc.

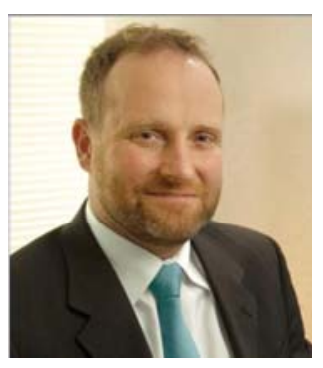

Chel Stromgren SAIC

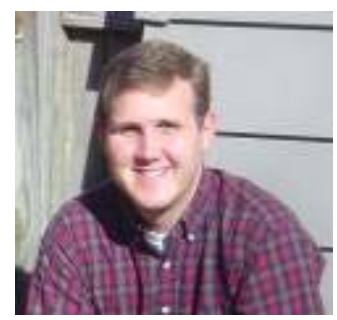

J. D. Reeves NASA LaRC

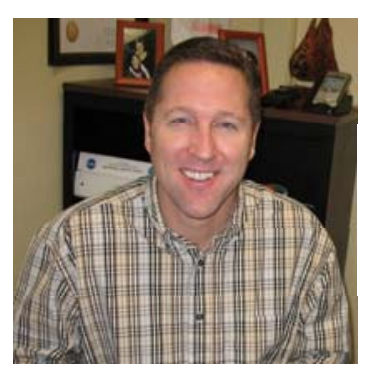

David North NASA LaRC 


\section{Backup}




\section{Comparison of Loss of Crew}

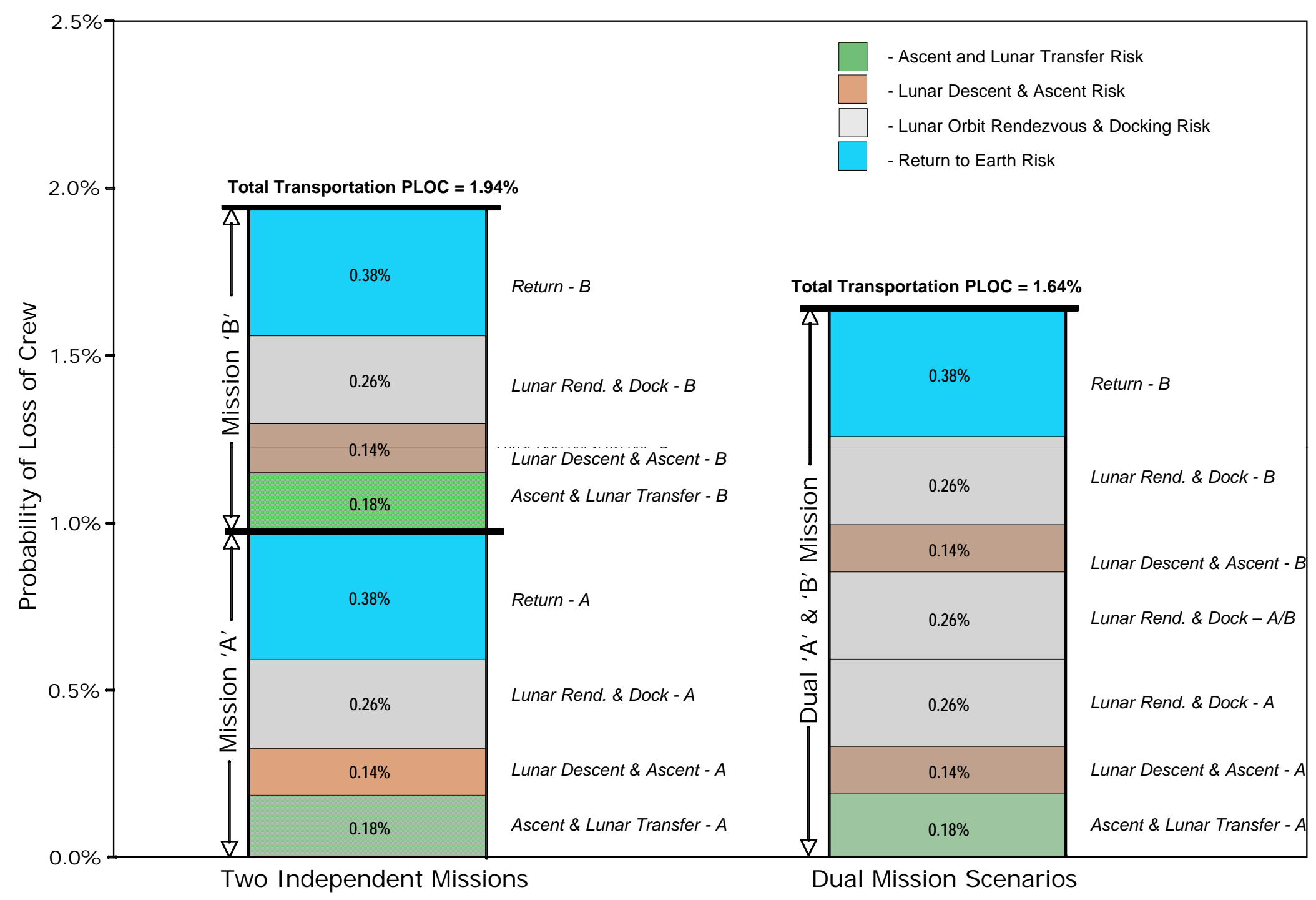




\section{Crew, 7 Day Loiter, 7 Day Stay}

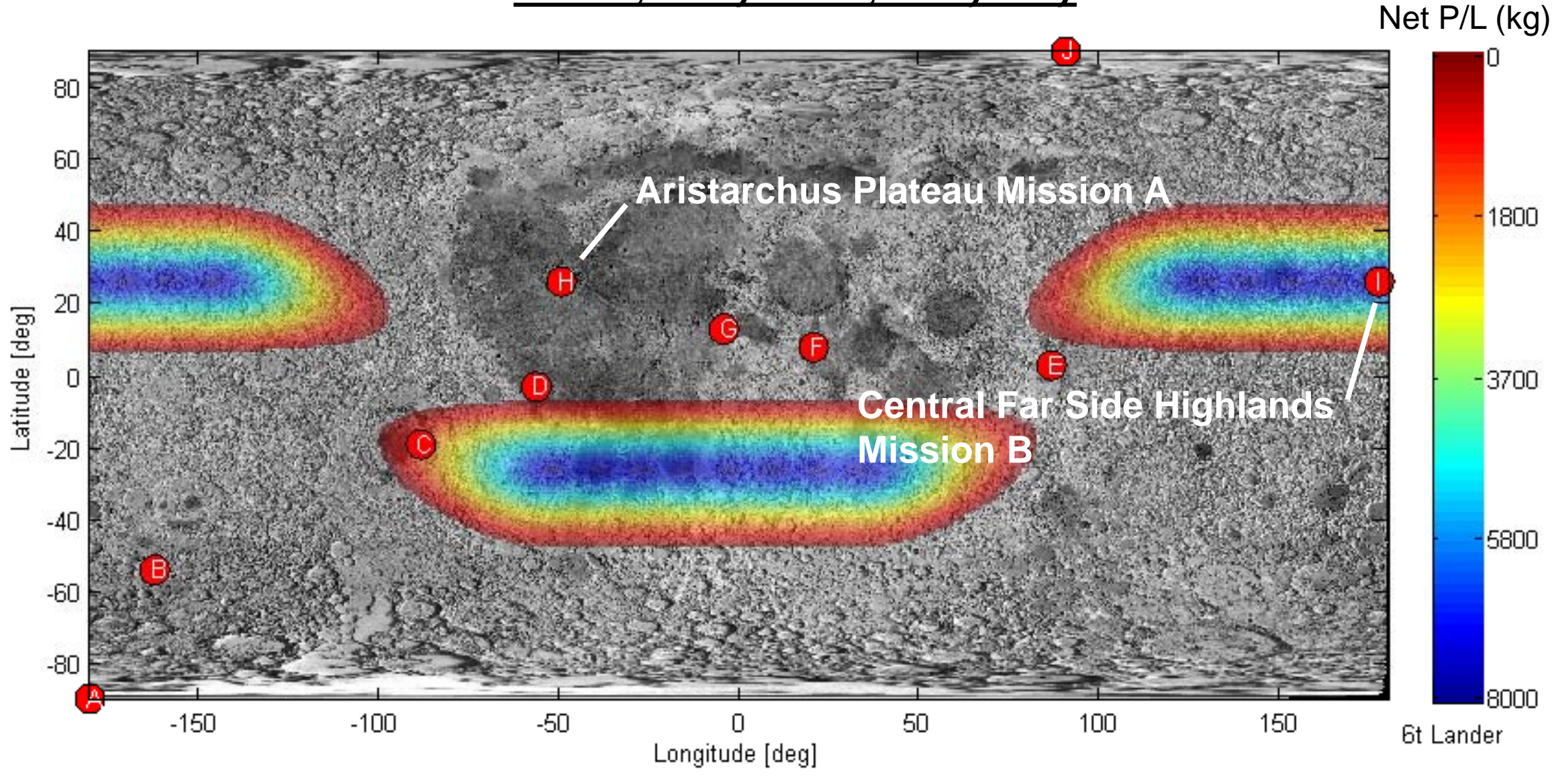

Mission B

Net $P / L(k g)$

\begin{tabular}{|c|l|c|c||c|l|c|c|}
\hline & \multicolumn{1}{|c|}{ Site } & Lat. & Long. & & \multicolumn{1}{|c|}{ Site } & Lat. & Long. \\
\hline A & South Pole & $89.9^{\circ} \mathrm{S}$ & $180^{\circ} \mathrm{W}$ & F & Mare Tranquillitatis & $8^{\circ} \mathrm{N}$ & $21^{\circ} \mathrm{E}$ \\
\hline B & Aitken Basin & $54^{\circ} \mathrm{S}$ & $162^{\circ} \mathrm{W}$ & G & Rima Bode & $13^{\circ} \mathrm{N}$ & $3.9^{\circ} \mathrm{W}$ \\
\hline C & Orientale Basin & $19 \mathrm{~S}$ & $88^{\circ} \mathrm{W}$ & H & Aristarchus Plateau & $26^{\circ} \mathrm{N}$ & $49^{\circ} \mathrm{W}$ \\
\hline D & Oceanus Procellarum & $3^{\circ} \mathrm{S}$ & $43^{\circ} \mathrm{W}$ & I & Central Far Side Highlands & $26^{\circ} \mathrm{N}$ & $178^{\circ} \mathrm{E}$ \\
\hline E & Mare Smythii & $2.5^{\circ} \mathrm{N}$ & $86.5^{\circ} \mathrm{E}$ & J & North Pole & $89.5^{\circ} \mathrm{N}$ & $91^{\circ} \mathrm{E}$ \\
\hline
\end{tabular}

* 2,709 kg Mission A payload to Aristarchus Plateau super sortie site;

$7,881 \mathrm{~kg}$ Mission B payload to Central Far Side Highlands sortie site. 